\title{
Intelligent COVID-19 Forecasting, Diagnoses and Monitoring Systems: A Survey
}

This paper was downloaded from TechRxiv (https://www.techrxiv.org).

LICENSE

CC BY 4.0

SUBMISSION DATE / POSTED DATE

$16-08-2021 / 18-08-2021$

\section{CITATION}

Anjum, Nasreen; Asif,, Amna; Kiran, Mehreen; Jabeen, Fouzia; Yang, Zhaohui; Huang, Chongwen; et al. (2021): Intelligent COVID-19 Forecasting, Diagnoses and Monitoring Systems: A Survey. TechRxiv. Preprint. https://doi.org/10.36227/techrxiv.15172488.v1

$\mathrm{DOI}$

10.36227/techrxiv.15172488.v1 


\title{
Intelligent COVID-19 Forecasting, Diagnoses and Monitoring Systems: A Survey
}

\author{
Nasreen Anjum, Amna Asif, Mehreen Kiran, Fouzia Jabeen, Zhahoui Yang, Chongwen Huang, Salma Noor, \\ Kanwal Imran, Ihsan Ali, Senior Member, IEEE, and Ehab Mahmoud Mohamed, Senior Member, IEEE,
}

\begin{abstract}
To date, the novel Corona virus (SARS-CoV-2) has infected millions and has caused the deaths of thousands of people around the world. At the moment, five antibodies, two from China, two from the U.S., and one from the UK, have already been widely utilized and numerous vaccines are under the trail process. In order to reach herd immunity, around $70 \%$ of the population would need to be inoculated. It may take several years to hinder the spread of SARS-CoV-2. Governments and concerned authorities have taken stringent measurements such as enforcing partial, complete, or smart lockdowns, building temporary medical facilities, advocating social distancing, and mandating masks in public as well as setting up awareness campaigns. Furthermore, there have been massive efforts in various research areas and a wide variety of tools, technologies and techniques have been explored and developed to combat the war against this pandemic. Interestingly, machine learning algorithms and internet of Things (IoTs) technology are the pioneers in this race. Up till now, several real-time and intelligent COVID-19 forecasting, diagnosing, and monitoring systems have been proposed to tackle the COVID-19 pandemic. In this article based on our extensive literature review, we provide a taxonomy based on the intelligent COVID-19 forecasting, diagnosing, and monitoring systems. We review the available literature extensively under the proposed taxonomy and have analyzed a significantly wide range of machine learning algorithms and IoTs which can be used in predicting the spread of COVID-19 and in diagnosing and monitoring the infected individuals. Furthermore, we identify the challenges and also provide our vision about the future research on COVID-19.
\end{abstract}

Index Terms-COVID-19, pandemic, Coronavirus, machine learning algorithms, Artificial Intelligence (AI), Internet of Things (IoTs), COVID-19 diagnoses, COVID-19 monitoring, COVID-19 forecasting.

N. Anjum, A. Asif, M. Kiran, F. Jabeen, K.Imran, and S.Noor are with the Department of Computer Science, Shaheed Benazir Bhutto Women University, Peshawar, Pakistan, (email address: Fist.last@sbbwu.edu.pk).

Z. Yang is with the Department of Electronic and Electrical Engineering, University College London, WC1E 6BT London, UK, (email: zhaohui.yang@ucl.ac.uk.)

C. Huang is with College of Information Science and Electronic Engineering, Zhejiang University, Hangzhou 310027, China, and with International Joint Innovation Center, Zhejiang University, Haining 314400, China, and also with Zhejiang Provincial Key Laboratory of Info. Proc., Commun. and Netw. (IPCAN), Hangzhou 310027, China. (e-mail: chongwenhuang@zju.edu.cn).

I. Ali is with Faculty of Computer Science and Information Technology, University of Malaya. (e-mail: ihsanali@ieee.org).

Ehab Mahmoud Mohamed is with Electrical Engineering Dept., Colleague of Engineering, Prince Sattam Bin Abdulaziz University, Wadi Addwasir, saudia Arabia, and also with Electrical Engineering Dept. Faculty of Engineering, Aswan University, Aswan, Egypt. (e-mail: ehabmahmoud@aswu.edu.eg).

\section{INTRODUCTION}

$\mathbf{F}$ IRST reported by officials in Wuhan, China, in December 2019, the SARS-CoV-2 infection had quickly spread by late January 2020 to every region of China and numerous different nations [1] [2] [3]. As of 21st January 2021, 213 countries and territories have registered COVID-19 cases. On January 30, 2020, the World Health Organization (WHO) declared that the outbreak constitutes a Public Health Emergency of International Concern (PHEIC); and on March 11th, the WHO announced the COVID-19 as a "global pandemic", and shortly after that, the United States declared COVID-19 outbreaks a national emergency [4] [5]. By April 6, 2020, more than $1,200,000$ individuals had been infected by the disease and, what is more, the number of deaths due to the Coronavirus exceeded 70,000 in over 213 nations. As of 18th Jun 2021, inspite of thorough worldwide regulation and isolated endeavors, the cases of COVID-19 keep on ascending globally, with $176,693,988$ research facility affirmed cases and more than $3,830,304$ deaths [6].

Due to this worldwide flare-up, more than 200 nations worldwide had initiated either a full lockdown or remote area lockdowns, affecting the lives of billions of people, directly and indirectly. COVID-19 has also significantly impacted the economy worldwide and has been more extreme than the 2008 global financial crises (GFC)] [7]. The stock exchanges have been imploded by half or more, credit markets have been frozen, a significant number of bankruptcies followed, jobless rates took off above $10 \%$, and GDP contracted at an annualized pace of $10 \%$ or more [8]. However, even though a few nations have forced 'smart lockdowns' in certain areas with potential COVID-19 cases, there has still been a flood of new COVID cases and an ascent in deaths [9] [10], worldwide due to the discovery of new COVID-19 stains in UK [11], South Africa [12], Brazil [13], Japan [14], USA [15] and India (Indian variant) [16]. It will cause the economy to contract again and markets will crash again.

Currently, five vaccines, two from China, two from the U.S., and one from the UK, have already been widely used for inoculation across the world, according to the WHO [17]. The first batch of the COVID-19 vaccine is now being distributed and in order to reach herd immunity, around 70 percent of the population would need to be vaccinated which might take upto a few years. Since developing world does not have access to vaccines, coronavirus will still ravage many parts of the world. Furthermore, according to the White House briefing [18], these vaccines are less effective against the new COVID- 
19 strains. Such an alarming situation would mean eradicating the virus may be impossible as new outbreaks emerge around the world and the potential for more contagious or virulent variants of SARS-CoV-2 becomes all the more dangerous. While vaccinations in combination with lockdown measures far and wide are forced to prevent new infections, it is not enough to stop the spread of COVID-19.

\section{A. Scope of the Survey Paper}

According to a research finding [19], new UK Corona strain is doubling the number of active cases every 10 days in the USA. Indian variant is $50 \%$ more contagious than the UK variant, which is already $50-100 \%$ more contagious and transmissible than the 'regular' strains [20]. In current conditions, intelligent, automated, and real-time systems which can estimate the Corona infection hotspot regions or the individuals who are already infected will give WHO and governments around the globe a useful tool to deal with the pandemic. Furthermore, intelligent tools and technologies that help in monitoring and diagnosing the COVID-19 is the need of today. Hence, the investigations of the novel COVID19 plagues, its future development pattern, fast and efficient monitoring and diagnoses system, have become the front line research points right now and have received a generous amount of consideration and attention from data scientists and researchers from all around the globe. This paper aims at conducting an extensive survey of the monitoring, forecasting and diagnoses systems proposed to fight against the COVID19 pandemic.

From the most recent 20 years a few pandemics have been accounted for, for example, acute respiratory syndrome Corona virus (SARS-CoV) in 2002 to 2003 [21] [22], H1N1 flu in 2009 [23] [24] [25] and the Middle East respiratory syndrome Coronavirus (MERS-CoV) in 2015 [26] [27] [28]. Accordingly, several intelligent and machine learning algorithms based forecasting systems have effectively been proposed and used to gauge the number of active cases, recuperated cases, deaths and the end date of the pandemic [29] [30] [30] [31] [27]. As these forecasting systems show better performance in predicting the spread rate of deadly diseases, the investigations of the novel COVID-19 plagues and its future development pattern has also received a generous amount of consideration and attention from data scientists and researchers from all around the globe. Researchers and data scientists have additionally utilized similar methodologies in the assessment of the spread rate and number of deaths due to the COVID-19 pandemic. As a first contribution, we presented an extensive survey of the forecasting models or systems proposed to estimate the spread rate of COVID-19 in different regions of the world.

Numerous studies have suggested the utilization of X-rays and Computed Tomography (CT-scans) for finding of COVID19 disease because of the restrictions and multifaceted nature of antigen and PCR tests [32] [33] [34]. As reported in [33], the detection of COVID-19 symptoms in the lower portion of the lungs has a higher accuracy when utilizing X-rays or CT-scans than that when utilizing PCR tests. In specific cases,
CT-Scan and X-ray tests can be subbed with RT-PCR tests. Nonetheless, because of high volume of infected individuals and predetermined number of radiologists, X-ray and CT-Scan techniques can not solely address the issue. Consequently, there is a gigantic interest in looking for alternative techniques that can moderate the pressure from the medical care facilities and help and assist the radiologists. Recently, machine learning algorithms have been recommended to tackle the issue. Therefore, as a second contribution in this survey article, we have discussed various COVID-19 diagnoses systems based on the machine learning algorithms proposed to diagnose COVID19 disease and victims using the chest $X$-ray datasets.

Internet of Thing (IoT) technology alongside its wearable sensor nodes and vision based technology (cameras) have effectively been utilized in monitoring and diagnosing constant illnesses, for example, patients experiencing Parkinson [35] and Alzheimer's [36] disease, monitoring of blood-glucose levels in diabetic patients [37], Respiratory rate [38], blood pressure [39] [40] [41] [42] [42] [43], and detecting cardiovascular failure $[44]^{1}$. Based on the advantages and the suitability of IoTs in healthcare systems, we have explored several research efforts towards developing IoT based COVID19 monitoring and diagnosis healthcare systems. Therefore, as a third contribution in this survey article, we have explored several research efforts towards developing IoT based COVID-19 monitoring and diagnosing healthcare systems.

\section{B. Comparison to other COVID-19 Survey Articles}

Since the COVID-19 outbreak (Dec-2019), a few published survey papers took a comprehensive review at the COVID19 pandemic from various perspectives. For example, the authors in [46] and [47] have distinguished potential uses of Artificial Intelligence (AI) tools and technologies that can be utilized in COVID-19 immunization planning and drug discovery. The authors in [48] introduced a broad survey of cutting edge AI devices in forecasting and finding numerous viral illnesses. Authors recommended that AI Tools can be utilized to gauge the structure of SARSCoV-2-related proteins, distinguish existing medications that might be repurposed to treat the infection, propose new compounds that might be promising for drug improvement, and recognize potential immunization targets. The authors in [49], discussed briefly uses of deep learning tools in diagnosing the COVID-19 infection. In [50], the authors provide a brief overview of the current status of IoT applications related to the Coronavirus, distinguish their deployment and operational challenges, and suggest potential opportunities to further contain the pandemic. The authors in [51] presented a comprehensive study on open source data sets and their applications in mitigating the COVID-19 transmission.

In contrast, to help the research community, to have an overall comprehension of the continuous exploration and potential research areas in COVID-19, our major contributions in this article are as follow:

\footnotetext{
${ }^{1}$ For more information on the applications of IoTs in healthcare systems the intrigued reader may refer to [45].
} 


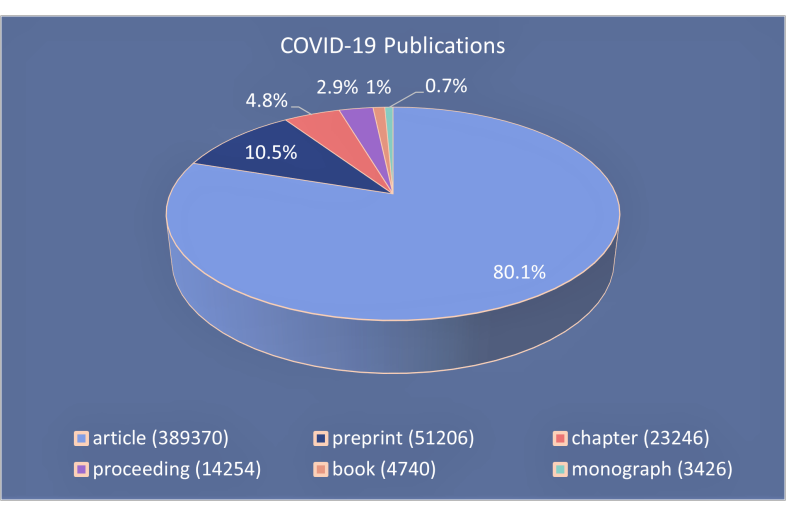

Fig. 1: COVID-19 publications worldwide [52].

\section{Contributions}

i. We provide an extensive review of a COVID-19 literature since the start of the COVID-19 pandemic. We have captured the most recent and highly cited research articles on machine learning algorithms and Internet of Things (IoTs) technology used to develop COVID-19 early detection, diagnoses, forecasting, and monitoring systems. Unlike the previous studies [46] [48] [49], which have only focused on general aspects of COVID19 related challenges in machine learning algorithms and IoTs, we have analyzed a significantly wider range of machine learning and deep learning algorithms. Focused is placed on data sets, tools, machine learning algorithms and IoT technology, which can be used in monitoring and diagnosing the COVID-19 symptoms and victims.

ii. Based on our extensive literature survey, we devise a taxonomy to characterize the COVID-19 intelligent and real-time systems into the forecasting, monitoring and diagnosing systems.

iii. We presented an extensive survey of the forecasting models or systems proposed to estimate the spread rate of COVID-19 in different regions of the world.

iv. We have discussed various COVID-19 diagnoses systems based on the machine learning algorithms proposed to diagnose COVID-19 disease and victims using the chest $\mathrm{X}$-ray datasets. Moreover, we have also surveys the role of IoT-based technologies in COVID-19 and reviews the best architectures, devices, and technologies for combating the Corona virus.

V. Finally, we identify the challenges and future directions and highlights the need to solve these issues.

\section{Paper Organization}

The rest of the paper is organized as follows. In Section II, we discuss the search strategy used to find the literature on COVID-19. Then, we introduce our taxonomy to survey the COVID-19 literature on intelligent forecasting, diagnoses, and monitoring system. We provide brief knowledge and background in machine learning in Section III. Then, several COVID-19 forecasting and diagnoses system based on machine learning algorithms are discussed in Section IV and Section V respectively. In Section VI background on IoT technology is presented and then several IoTs-based architectures, devices, tools, and technologies are reviewed in details used in monitoring the COVID-19 victims. Finally Section VII concludes our research efforts.

\section{INTRODUCTION TO SEARCH STRATEGY AND TAXONOMY}

In this section, we first discuss the search strategy used to find the related literature on COVID-19. Then, we introduce our proposed taxonomy for surveying the COVID-19 intelligent forecasting, diagnosis, and monitoring system. We likewise discuss the advantages of each system for the readers' interest and knowledge.

\section{A. Search Strategy}

According to Dimension Database [52], As of 23rd July 2021, scientists from 26,652 organizations have published over 569,979 articles about the corona virus (Fig. 1). Among which, over 7,969 included the phrases machine learning", artificial intelligence", deep learning", or neural network" etc, in the title or abstract. However, we emphasize that the search results may vary and depend on the reference and keyword search.

In this research study, legitimate databases, including IEEE Xplore $^{2}$, ScienceDirect ${ }^{3}$, ELSEVIER Chaos, Solitons \& Fractals ${ }^{4}$, SpringerLink ${ }^{5}, \mathrm{ACM}^{6}$, and ArXiv ${ }^{7}$, NATURE, PUBX, have been utilized to look for COVID-19 papers. Besides, a more definite Google Scholar ${ }^{8}$ search was utilized. The articles are chosen utilizing the keywords COVID-19, Corona virus, deep learning, machine learning, Artificial Intelligence, COVID-19 forecasting systems, COVID-19 diagnosis systems, IoTs, and COVID-19 monitoring systems. The most recent selection of papers is finished with the referenced keywords on 31 July, 2021.

\section{B. Taxonomy}

To this end, we identify the main applications of machine learning algorithms and IoT technology in predicting, diagnosing, and monitoring the COVID-19 victims and disease. Based on our extensive literature review, we organize the literature into three different groups. In the following section, we provide a brief introduction and advantages for each category. Fig. 2 shows the taxonomy we proposed.

1) COVID-19 Forecasting Systems: As a result of numerous advantages of machine learning approaches in forecast of viral diseases such as influenza [53], a seasonal flue in USA, many research efforts have distinguished machine learning techniques as a viable answer to forecast the COVID-19 areas and infected people. In several works, prediction models have been developed for explicit purposes for instance, for forecast of number of confirmed cases, number of recovered cases, number of deaths, and more.

\footnotetext{
${ }^{2} \mathrm{https} / / /$ ieeexplore.ieee.org/

${ }^{3} \mathrm{https}: / /$ www.sciencedirect.com/

${ }^{4}$ https://www.journals.elsevier.com/chaos-solitons-and-fractals

${ }^{5}$ https://link.springer.com/

${ }^{6}$ https://dl.acm.org/

${ }^{7}$ https://arxiv.org/

${ }^{8}$ https://scholar.google.com/
} 


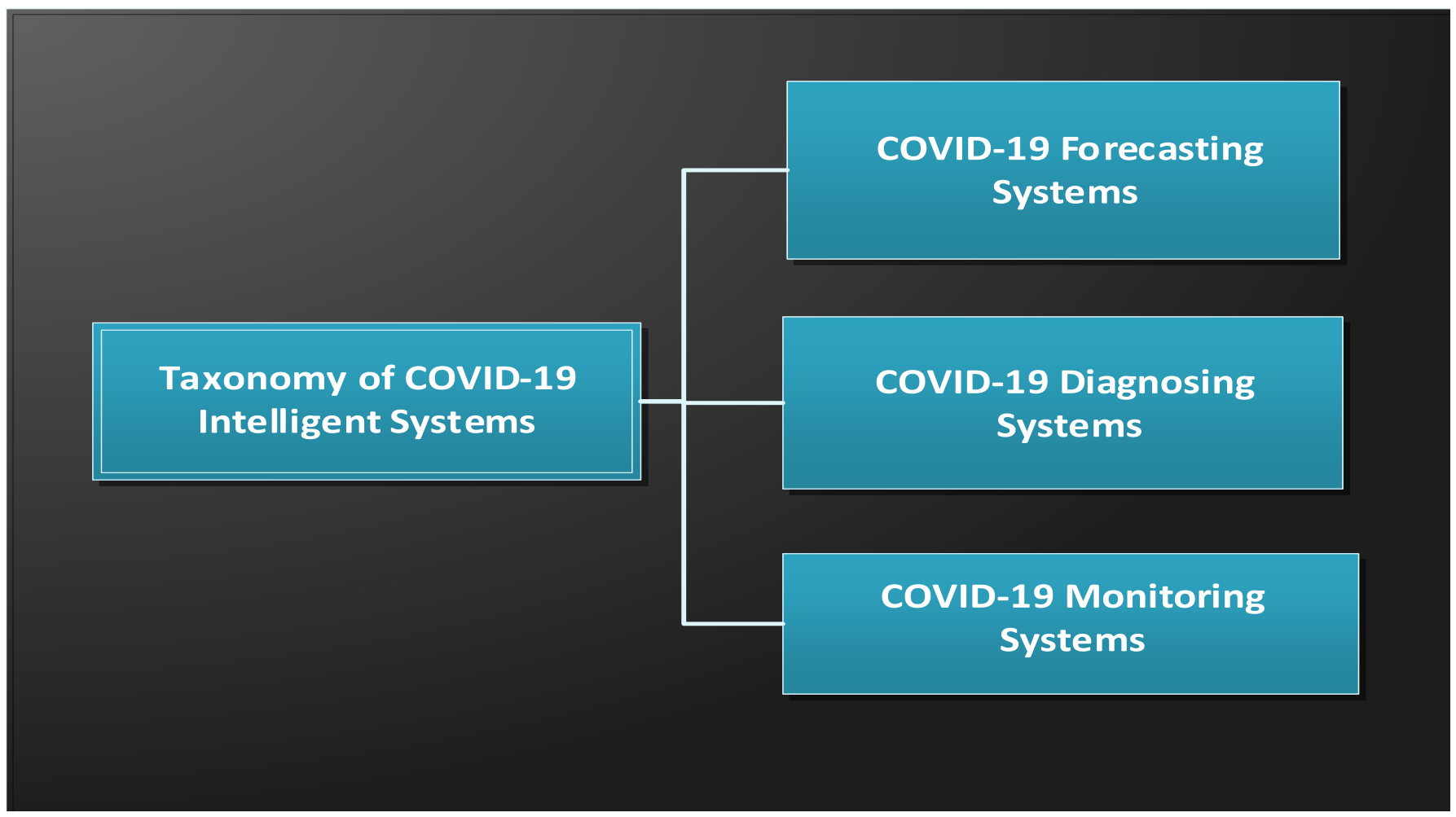

Fig. 2: Taxonomy for surveying the intelligent COVID-19 forecasting, diagnoses, and monitoring systems.

As a first contribution, this paper presented an extensive survey of the forecasting models or systems proposed to estimate the spread rate and death rate of COVID-19 in different regions of the world.

Benefits of COVID-19 Forecasting Systems: The intelligent COVID-19 forecasting frameworks based on machine learning algorithms are integral assets to minimize the effect of Corona virus in specific areas by predicting the regions where the number of infected individuals is high. By predicting the areas of high vulnerability, individuals can get a fair warning and take action. They are given awareness and warning to maintain a 6 feet distance from others and to wear masks while in public places [54] [55]. This will decrease their chances of contracting the virus. Decreasing the risk of contracting the virus won't just save lives, however will likewise prevent the infection from spreading. It can also give a warning the government to take action within that area. The concerned health authorities can impose a partial, complete, or smart lock down in that area and restrict the movement of individuals from within that area. Moreover, local authorities can plan a public health infrastructure to reduce the unprecedented pressure on hospitals [56] like number of beds, staff, ventilators and other health equipment required beforehand.

2) COVID-19 Diagnosing Systems: The literature under this category, discussed various machine learning algorithms for exploiting the diagnoses of COVID-19 through the chest $\mathrm{X}$-ray and CT-Scan.

Benefits of COVID-19 Diagnosing Systems: Machine learning algorithms can assist in the clinical decision making and have been used successfully in diagnosis of a number of health problems such as pathological brain detection [57] [58], breast cancer [59], lung cancer [60] [61], colon cancer [62] [63], prostate cancer [63], Alzheimer's disease [64], diabetes [65] and flu [66]. This entails the use of intelligent approaches that can automatically extract useful insights from the chest X-rays those are characteristics of COVID-19.

The main aim is to lessen the time and exertion needed to perform CT-Scan and X-rays of COVID-19-positive patients and assess the pace of disease development [32] [33] [34]. Furthermore, these results can be covey to healthcare physicians, who would then be able to react quickly to suspected cases and carry out the necessary examinations to confirm the case. This permits the confirmed cases to be isolated and given appropriate health care.

3) COVID-19 Monitoring Systems: The literature under this category, has explored the suitability of IoT technology in COVID-19 pandemic. We have discussed numerous research efforts towards developing IoT based COVID-19 monitoring and diagnosis healthcare systems.

Benefits of COVID-19 Monitoring Systems: An IoT based monitoring and diagnosis system can help in hindering the spread rate of the COVID-19 virus. The wearable sensors can help in monitoring, as well as diagnose the symptoms of COVID-19 disease, for example, body temperature, coughing patterns, and blood oxygen levels. These sensors with the assistance of AI techniques can issue a clear warning to the potentially infected individual and the concerned authority to take precautionary measurements, for example, to isolate themselves and to take appropriate tests and avoid social gathering. With the assistance of geo-area services and different technological advancements, for example, Bluetooth etc., individuals can be cautioned through the alarm on the off chance 
that they come close to another individual. Governments and health specialists may utilize these platforms and information for permanent surveillance after a pandemic to control and track individuals' behaviors.

One of the significant advantages of IoTs in health care system is remote monitoring [44]. For instance, SPHERE [44] is a framework under continuing development that uses wearable, environmental, and vision-based (for example, camera) sensors for general activity and health monitoring purposes. Further, remote monitoring has been utilized effectively in hospitals by doctors, to monitor the non-critical patients or elderly individuals at home [67]. Likewise, IoTs technology can be be very useful to monitor the COVID-19 patients remotely those who are in isolation wards. Moreover, the family of the patient can keep an eye on the COVID-19 patients who are quarantined at home. It tends to be valuable in monitoring infected patients at who are quarantined at home or isolated elsewhere. Essentially, it can improve access to healthcare resources whilst reducing the strain on healthcare frameworks, and can give individuals better control over their own wellbeing at all times. Artificial intelligence assisted sensors can be utilized to help predict whether individuals are contaminated with the infection, in view of symptoms, such as body temperature, coughing patterns, and blood oxygen levels. IoT technology can help in contact tracing and restricting an individuals access to public places through applications and platforms empowered by AI.

\section{BACKGROUND ON MACHINE LEARNING Algorithms}

Over the decades, AI has been evolved in two distinct and parallel directions: machine learning and expert systems. Focusing on first, machine learning enables machines to make accurate predictions through utilizing vast data sets, perform clustering, extract association rules, or make decisions from a given data sources. To archive the learning process, computer systems are trained using the various algorithms and statistical models. Some of the most popular machine learning applications are traffic alerts and smart transportation [68], social media alerts such as automatic friend tagging in Facebook [69], products recommendations [70], fraud detection [71], weatherforecasting [72] [73] [74], and stockmarket forecasting [75] [76].

There are four machine learning methods ${ }^{9}$ : supervised, unsupervised, semi-supervised, deep learning, and reinforcement learning Fig. 3. Our literature review shows that a majority of research papers have used these machine learning algorithms and their variants in predicting and diagnosing the corona disease. In this section, we will focus on the machine learning algorithms which have been applied to Corona disease datasets to diagnose and predict the spread of Corona virus.

\section{A. Supervised Machine Learning Algorithm}

A supervised machine learning algorithm takes a known set of input data, for instance, Corona disease symptoms

\footnotetext{
${ }^{9}$ For reader convenience, we only focus on ML algorithms used for predictive analysis. For more information on ML algorithms the interested readers are refer to [77] [78].
}

such as, cough, body temperature, oxygen saturation, and age etc., and known responses to the data, for instance, number of infections, deaths, recovered etc., and trains a model to generate reasonable predictions for the responses to new data. Supervised machine learning algorithm works in two phases: (a) training phase and, (b) testing phase. The training phase learns on labeled data set. The testing phase checks the validity of results [79]. The supervised machine learning algorithms find the relationship between the input values and labels and try to predict the appropriate output values of the testing data. Supervised machine learning algorithms are popular for solving the regression and classification problems. Therefore, they are categorized as regression model and classification model.

1) Regression Model: The supervised ML algorithms fall under the regression category are capable of predicting a result based on the previously provided data set and the obtained results from them. Weather forecasting [80], stock market forecasting [81], population growth forecasting, products recommendations [70], and fraud detection [71] are the most popular applications of supervised regression algorithms. Due to the advantages of regression algorithms in predictive analysis, several researchers have used regression algorithms such as linear regression, non-linear regression, multi-linear regression, to forecast the spread pattern of corona disease. Brief introduction of some of the popular regression models is given below:

a. Linear Regression (LR): Linear regression is a well-known, simple and tremendously powerful technique for the prediction of the value of a variable called the response/output/ dependent variable given the value of another variable called explanatory/input/independent variable [82]. They are called linear because they show a linear relationship between a dependent and one or more independent variables. Based on the number of independent variables linear regression is further classified as Simple Linear Regression (SLR) and Multiple Linear Regression (MLR). SLR make use of single independent variable to predict the value of a numerical dependent variable, while MLR use more than one independent variables to predict the value of a numerical dependent variable. Linear regression makes predictions for continuous/real or numeric variables such as sales, salary, age, product price, rainfall intensity etc., using the historical data.

b. Non-Linear Regression (NLR): Sometimes linear models are not sufficient to capture the real-world phenomena, and thus nonlinear models are necessary and used as alternative solutions. It is used in place when the data shows a curvy trend. One example of a nonlinear regression is a scatter plot of changing population data over time.

2) Classification Model: The regression model and classification model both share the same concept. The only difference is, the output variable in regression is 


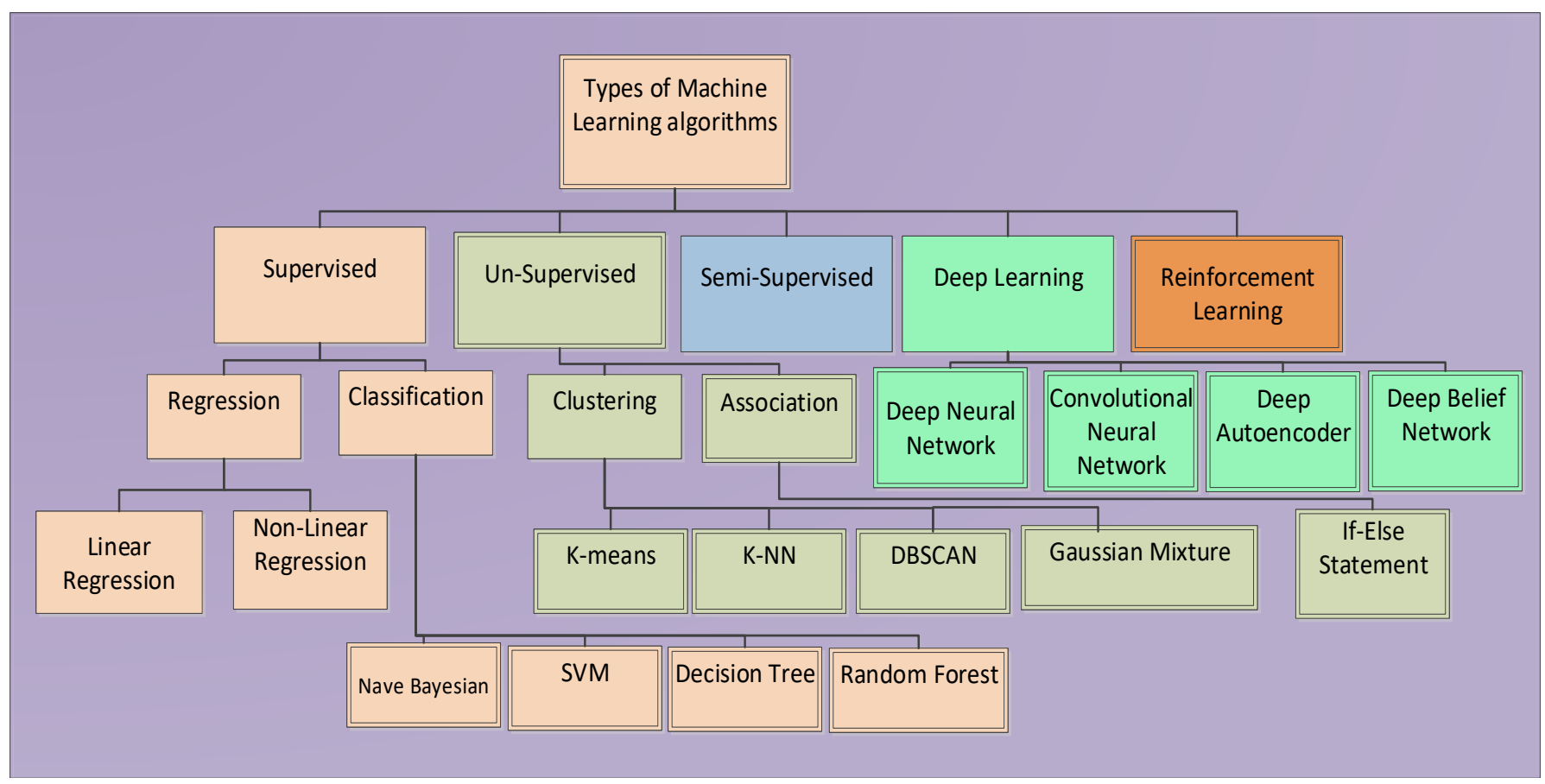

Fig. 3: Types of Machine Learning Algorithms.

numerical (continuous), while that for classification is categorical (discrete). For example, when provided with a patient's data set about Corona disease symptoms, a classification algorithm can try to predict whether the patient "is Corona positive" or "no Corona positive" (Negative)", "disease" or "no disease". Examples of the common classification algorithms include Naïve Bayesian model, Support Vector Machines (SVM), decision tree, and random Forest.

Naïve Bayesian model is very popular classification model as it is easy to build and produce more accurate results for large datasets. SVM and random forest algorithm both are very powerful supervised machine learning algorithms and can be used for both regression and classification problems. The most popular applications of SVM algorithm is the classification of images or text. Random Forest algorithm is popular for finance and business applications. For example, it is used to predict whether customers will repay their debt on time or not, or will use a bank's services more frequently or not, or a customer will buy the product or not. In trading, the algorithm can be used to determine a stock's future behavior.

\section{B. Unsupervised Machine Learning Algorithms}

Unsupervised machine learning algorithms are used for decision making from data sets constitutes of unlabeled data such as a data set comprising of set of images. In comparison to supervised machine learning algorithms, unsupervised machine learning algorithms can help users to solve more complex problems such as in identifying hidden patterns which were previously unknown or undetected, in finding the features of some product or object that can be useful for classification, clustering or group the data, and in predicting the future values [83]. Moreover, unsupervised machine learning algorithms are utilized for clustering problems such as recommendation systems, customer segmentation, and targeted marketing. Unsupervised learning problems can be further grouped into clustering and association problems.

1) Clustering Algorithms: Several research efforts have used clustering algorithms to forecast the COVID infected areas and categorize the regions. Clustering algorithms help in finding the hidden grouping in data based on the similar character or features of data such as grouping regions with high number of corona patients. K-means [84], K-NN (k nearest neighbors) [85], DBSCAN (Density-Based Spatial Clustering of Applications with Noise) [86], Gaussian Mixture Model [87] and spectral clustering [88] etc., are the most popular and well known clustering algorithms.

2) Association Algorithms: Association algorithms helps in finding an association or relationship among the data points in a large database. For example, a subgroup of corona patients grouped based on their symptoms and laboratory reports. Association rule learning works on the concept of If and Else Statement, such as if a patient has a symptoms A, Symptom B, and Symptom C then he/she is more likely to have corona disease.

Limitations of Supervised and Unsupervised Machine Learning Algorithms: The most basic disadvantage of any supervised machine Learning algorithm is that, the data set has to be labeled manually either by a machine learning engineer or a data scientist. This is a very costly process in terms of time and fatigue, especially when the size of data-set is very large. Likewise, unsupervised machine learning algorithms are considered as the real-world applications limited algorithms. 


\section{Semi-Supervised Machine Learning Algorithms}

To deal with the above mentioned disadvantages of supervised and unsupervised machine learning algorithms, the concept of semi-supervised learning was introduced.

Semi-supervised machine learning algorithms are the extension of both supervised and unsupervised machine learning algorithm or the combination of both algorithms. The semisupervised machine learning algorithm trains on a data set that contains both labeled and unlabeled data, but the amount of unlabeled data is much more than the labeled data.

Semi-supervised Learning methods are available in many flavors such as Semi-supervised classification, Constrained clustering, regression with labeled data and regression with unlabeled data. "Semi-supervised classification" is an extension to the supervised classification problems. The goal of semisupervised classification is to train a classification algorithm from both the labeled and unlabeled data instead of train on the label data alone. "Constrained clustering" is an extension to the unsupervised clustering. The training data consists of unlabeled instances as well as some "supervised information" about the clusters. The goal of constrained clustering is to obtain better clustering than the clustering from unlabeled data alone. Some popular applications of semi-supervised machine learning algorithms are Speech Analysis [89], Internet content classification [90], and Protein Sequence Classification [91].

\section{Deep Learning}

Deep Learning is the sub-field of AI that mimics the human brain functions and extracts abstract features from a large unstructured or unlabeled training data sets without any human supervision. Deep learning techniques can perform image, sound, or text classifications with the state-of-the-art accuracy and sometimes beyond the human level perfections. Deep learning techniques on chest X-Rays and CT-Scan images are getting popularity. Several deep learning architectures including deep neural network (DNN), RNN, convolutional neural network (CNN), deep autoencoder (DA), deep Boltzmann machine (DBM), deep belief network (DBN), deep residual network, deep convolutional inverse graphics network, have been reported to be benifical in solving the biomedical health problems such as brain tumor detection [92] [93], breast cancer detection [94], and so on. Convolutional Neural Network $(\mathrm{CNN})$ is the most popular deep learning algorithm. It is a multilayer neural network comprising of an input layer, hidden layers and an output layer [95]. inspired by the neurobiology of visual cortex, CNN has been proven extremely beneficial in feature extraction and image analysis. Some studies shows that CNN shows better performance for a large training datasets [96]. AlexNet [97], VGGNet [98], and GoogLeNet [99] are the most popular extensions of $\mathrm{CNN}$.

\section{E. Reinforcement Learning}

Reinforcement learning is different from both supervised and unsupervised machine learning algorithms. In this learning style, the algorithms try to predict the output for a problem based on a set of tuning parameters. Then, the calculated output becomes an input parameter and new output is calculated until the optimal output is found. In simple words, reinforcement learning algorithms enable systems to learn from the experiences they get through interacting with the environment. Reinforcement learning is mainly used for applications like AI gaming, skill acquisition, robot navigation, Natural Language processing and real-time decisions [100] [101].

\section{COVID-19 ForeCASTING Systems}

In this section, we presented an extensive survey of the forecasting models or systems proposed to estimate the spread rate of COVID-19 in different regions of the world. Table I has summarizes the combined findings from the reviewed literature, presenting the algorithm name, Benefits of proposed forecasting algorithms, data, time year and country for which the algorithm is proposed. While these prediction systems have been developed for estimating the distinctive COVID19 variables, they are firmly related through their utilization of similar machine learning techniques.

A forecasting system, named Augmented ARGONet, is developed to predict COVID-19 confirmed cases in China using the clustering (Auto regression) and data augmentation [116] techniques [102]. The dataset used to develop and train the prediction model was built using the data gathered from the internet, news alerts, and daily forecasts from health officials. The accuracy and reliability of proposed model are measured against the forecasts obtained from the sources such as persistent model and auto regressive model. Authors claim that Augmented ARGONet has the ability to predict the number of positive cases two days ahead of the current time and outperforms the auto regressive model.

In [103], clustering and modified auto encoder techniques are used to predict the COVID-19 confirmed cases, duration and ending time of COVID-19 outbreak across the China. Based on the conclusions obtained from their proposed forecasting model, authors claim that COVID-19 pandemic will be over by April, 2020 from all around the China. Furthermore, authors suggest that the increase in training time of prediction algorithms could lead to timely, reliable, and more accurate COVID-19 prediction systems.

The authors in [105] developed COVID-19 forecasting systems to estimate the COVID-19 spread including number of positive cases, recovered cases and deaths during the lock down period in India. Based on the predictions of forecasting models, authors proposed that strict lock down can significantly reduce the number of active cases from India. They utilized non-linear regression, decision tree, and random forecast model to compare the prediction outcomes and select the best forecasting model. The authors, from their results, proposed that random forest Model outperforms then the other machine learning models. Similarly, the authors in [104] used the linear regression, clustering and LSTM (Long short-term memory: a deep learning model) to forecast the peak in COVID-19 positive cases in India.

The COVID-19 virus infects individuals of all ages. Nonetheless, evidence to date suggests that older individuals and those with underlying critical medical conditions are at a higher risk of getting serious COVID-19 illness [117]. 
TABLE I: COVID-19 Forcasting Systems

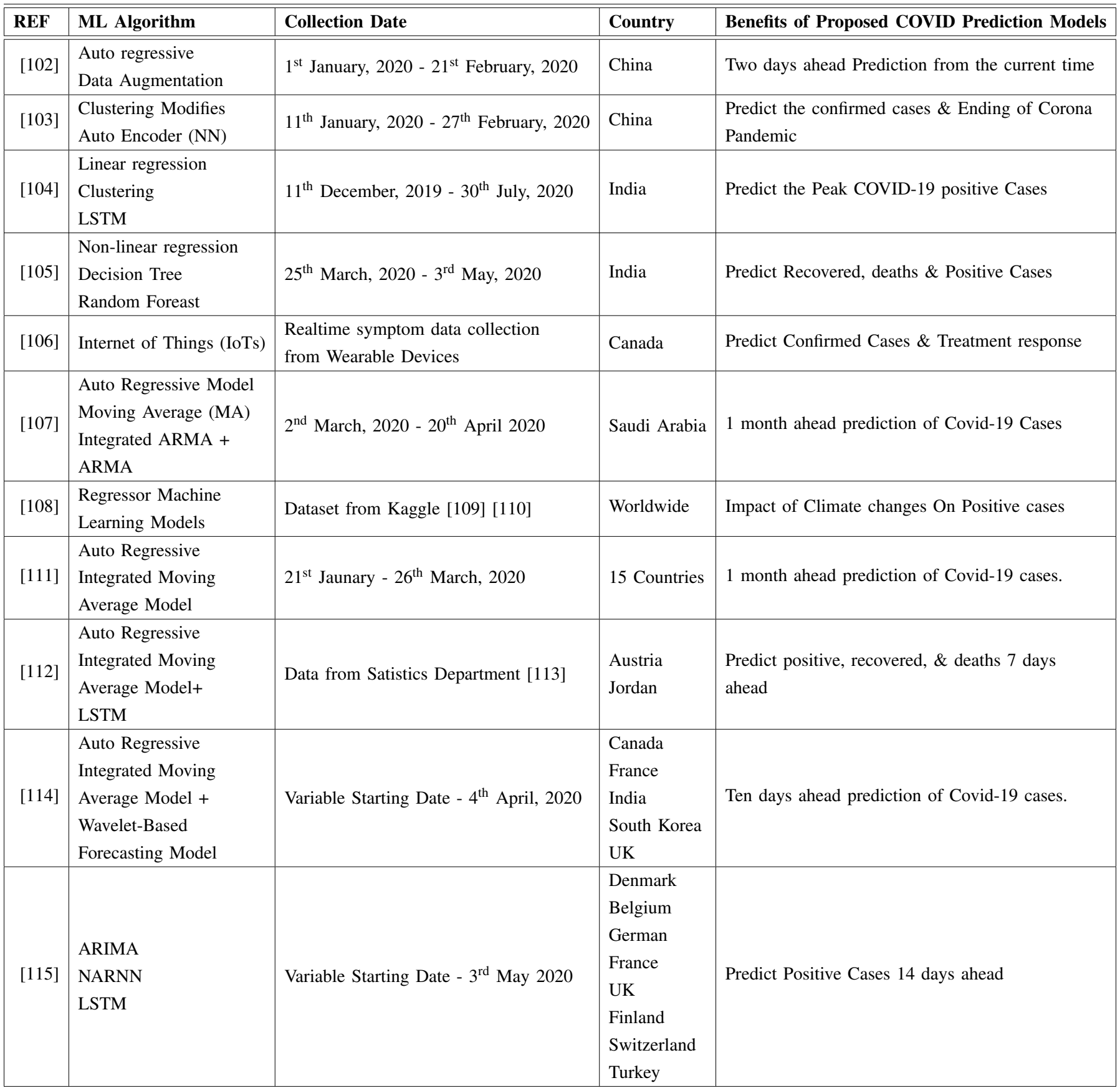

While considering age as a critical parameter, authors in [114] proposed a hybrid prediction model that combines the auto-regressive integrated moving average model and Waveletbased forecasting model [118]. Authors claim that this model can forecast the number of affirmed cases ten days ahead. The authors additionally investigated the affects of age on fertility rate for Canada, France, India, South Korea, and the UK. The authors suggest that by considering parameters, for example, number of confirmed cases, individuals of age group $>65$ years, lock down period, hospital beds per 1000 individuals) the respective nation may reduce the number of casualties at a significant rate.

The authors in [107] developed four diverse intelligent
COVID-19 forecast systems to estimate the number of positive cases in: Saudi Arabia, a month ahead. Autoregressive (AR) Model, Moving Average (MA), a combination of both (ARMA), and integrated ARMA (ARIMA) are utilized and ARIMA model was found as the best forecast model. Considering the results obtained, the authors suggest that without the solid measurements and limitations set against the COVID, the specialists of Saudi Arabia may see later in the future, 668 new cases each day and more than 127,129 cumulative daily cases. Similarly, authors in [111] predict the number of positive cases and deaths in 25 nations including Iran, UK, European countries, and China. In light of the results of the predictive model, authors claim that USA will be the most affected 
COVID-19 area in the world in Apil 2020. Authors in [112] utilized the ARIMA and Long short tem memory (LSTM) model to predict the number of positive cases, recoveries, and deaths in Jordan and Australia. The authors claim that the prediction model has the capability to anticipate Coronavirus spread 7 days ahead.

In [115], potential COVID-19 cases in Denmark, Belgium, Germany, France, United Kingdom, Finland, Switzerland and Turkey were modeled utilizing the ARIMA, Nonlinear Auto regression Neural Network (NARNN) and Long-Short Term Memory (LSTM) approaches. LSTM was demonstrated to be the most accurate model and claimed to forecast 14-days ahead.

In [106] machine learning algorithms in combination with Internet of Things (IoTs) technology [45] are used to develop the COVID-19 forecasting system. Their work concludes that wearable sensors for observing and noting COVID-19 symptoms in individuals such as fever, cough, fatigue, sore throat, and shortness of breath could be used in combination with machine learning algorithms to build an effective and efficient forecasting system. They used and compared the performance of eight machine learning algorithms, namely Support Vector Machine, Neural Network, Naïve Bayes, K-Nearest Neighbor, Decision Table, Decision Stump, OneR, and ZeroR. Their findings suggest that five ML algorithms have the potential to predict COVID-19 positive cases with $92 \%$ accuracy.

The authors in [108] presented a COVID-19 forecasting system while taking into consideration weather conditions such as temperature and humidity. Based on the predictive analysis, a strong correlation was found between temperature, COVID-19 spread, and mortality rate. Authors illustrate that the higher the value of temperature, lower the number of infection cases. we have proved that climatic conditions such as temperature and humidity contribute to the spread of the virus. Similarly, authors in [119] have illustrated that the virulence of coronavirus diseases due to viruses such as SARS$\mathrm{CoV}$ and MERS-CoV decrease in humid and hot weather climatic conditions.

\section{A. Challenges and Lesson Learned}

In this section, we thoroughly reviewed the intelligent COVID-19 forecasting systems proposed and developed to forecast the spread rate of COVID-19 in different regions of the world including Chine, India, USA, Saudia Arabia, Australia, South Korea and many European countries. From this section we learned the importance of machine learning algorithms in COVID-19 pandemic. We found that, machine learning algorithms along their variants are extremely important and are powerful tools in forecasting the future transmission rate of corona virus and any future pandemic. Moreover, They can be precious assets for the government and healthcare authorities to plane their strategic policies to deal with the COVID-19 outbreak.

A variety of parameters have been considered while building these models such as number of confirmed cases, number of deaths, weather conditions, and age etc. However, many parameters have been ignored while designing these prediction systems which are playing their crucial part in transmission of this disease such as type of COVID-19 strain in the contaminated area, travel history of individuals, Vaccinated or not vaccinated, age, health history of individuals, and diet, etc. Indian variant (also called Delta variant) is more contagious and can infect 50 percent more population then the regular strain. Likewise, mortality rate is higher for age group above 50 and in individuals with chronic health history. Therefore, we suggest to broader the decision parameter range to obtained accurate and reliable results.

Machine learning techniques based on supervised, unsupervised, and deep learning algorithms provides the opportunity to develop a medical diagnosis systems. However, we found that, developing an accurate COVID-19 predictive model is a challenging task due to multiple reasons. For instance, to produce reliable and accurate results, machine learning algorithms require a substantial amount of training data. Due to unavailability of up-to-dated health records, under-reporting COVID19 cases, scarce availability of epidemiological information about COVID-19 virus (as there is no historical information about the behavior of this disease is available), emerging new Corona strains and related array of uncertainties of COVID-19 disease, currently available datesets lacks correct and sufficient amount of information. As a consequent they are hindering the efficiency of machine learning algorithms and $100 \%$ reliable and accurate prediction results can not be achieved. To enrich the training datasets with valuable, correct, and huge information many serious steps need to be taken. For instance,

- a collaboration among health officials (Doctors, nurses, microbiologists, pharmaceutical companies, clinicians) and IT experts (data scientists, computer scientists, engineers) is mandatory to facilitate this advancement and actual the need of today to deal and manage the upcoming waves of COVID-19 pandemic and any future pandemic.

- In general, the cause of under-reporting data is a privacy concern of patients. A majority of patients are not willing to share their health data publicly on some remote centralized storage media such as cloud or edge servers. These centralized storage systems are managed and owned by the private organizations, which might lead to loss, unavailability, and misuse of patient's data. Under such situation, decentralized security approaches such as block-chain technology can be adopted to avoid the security concerns of patients and cloud-IoT platforms owned only by authorized entities, such as hospitals, government, or other highly trusted entities through some private cloud need to be developed. Furthermore, consent for safe data sharing and privacy agreements may be require to gain the trust of the patients.

\section{COVID-19 Diagnosing Systems}

In this section we first discuss the available COVID-19 diagnostic tests and their limitations. Then we discuss different machine learning algorithms for exploiting the diagnoses of COVID-19 through the chest X-ray and CT-Scan. The research contributions in this area are summarized in Table II. 


\section{A. Traditional COVID-19 Diagnosing Tests}

A wide range of diagnostic tests are available to decide whether or not somebody has the active COVID-19 disease? These symptomatic tests fall into two classes [120] [121]:

i. Antigen tests or Rapid-Point-of-Care-Test: They are taken with a nasal or throat swab and detect a protein that is a part of the corona virus [122]. These tests are particularly conveniant for identifying an individual who is at or near peak infection. Antigen tests are less expensive and faster, as they do not need complex and expensive test kits to detect the antigen. The drawback is that they can be less accurate [123]. This means, if an individual is not near peak infection - but is still highly contagious - the tests may come back as negative. Depending on the quality of the antigen test and the test takers, false negatives could account for $20 \%$ [121].

ii. Molecular or PCR tests: The Centers for Disease Control and Prevention (CDC) has exhorted individuals who show COVID-19 indications yet test negative with a fast antigen test to get a PCR test to affirm the results. Molecular tests distinguish hereditary material - the RNA - of the COVID19. The best PCR tests commonly require trained faculty, specific reagents and higher cost (about 120-130 USD \$). The sample is gathered with a nasal or throat swab and they will in general require hours to give results. Great PCR tests are nearly $100 \%$ precise.

\section{Limitations of Traditional COVID-19 Diagnosing} Tests

Notwithstanding, not every molecular test, including PCR tests, are perfect. Some low quality testing platforms have detailed false negative rates as high as $15 \%$ to $20 \%$. The limited availability of the RT-PCR test kits makes it challenging to detect every individual affected by the virus. Furthermore, these tests take from few hours to a day or two to produce the output, which becomes too tedious, time consuming and most of the time error prone in the current state of emergency.

\section{B. Machine Learning Algorithms Based on COVID-19 Diagnosing Systems}

To diagnose the COVID-19 victims based on the chest $\mathrm{X}$-ray images, in [124] an automatic diagnostic system based on deep CNN, named COVID-Net, is presented. The proposed model is trained on their own created open access data set COVIDx. COVIDx comprises of 13,975 X-ray images of 13,870 COVID-19 patients. The authors claimed a higher test accuracy and COVID-19 sensitivity for COVID-NET against the VGG-19 and ResNet-50 network architectures. For instance, COVID-19 sensitivity of COVID-Net is $32 \%$ higher than VGG-19 and $8 \%$ higher than ResNet-50 [147].

The authors in [126] utilize the pretrained ResNet50 architecture and further improve COVID-NET overall accuracy to $96.23 \%$. Authors claimed that their proposed
COVID-19 diagnosing system, named COVID-ResNet, has better performance in terms of speed, accuracy, and computation. The reason illustrated by the authors as to why COVID-ResNet shows better performance is because ResNet-50 is build on a less number of layers and parameters than the conventional $\mathrm{CNN}$ architecture.

The authors in [33] also recommended ResNet50 over other four models including ResNet101, ResNet152, InceptionV3 and MobileNet v2. Authors trained these models using the three datasets collected from open source GitHub database [127], Kaggle repository, and a database consisting of healthy chest X-ray images [128]. All datasets comprises of 341 chest X-ray images of COVID19 patients. It is claimed that ResNet50 achieved over 95\% accuracy among four other models.

Due to the unavailability of large dataset of COVID-19 positve X-ray images, the authors in [130] utilize the transfer learning approach to evaluate the performance of deep CNN architectures (VGG19, MobileNet v2, Inception, Xception, Inception ResNet v2) in diagnosing the COVID-19 disease from the chest X-ray images. The authors claim that use of transfer learning approach on small datasets can produce remarkable results. Furthermore, experimental results show that in terms of classification accuracy VGG19 and the MobileNet v2 outperforms over the Inception, Xception, Inception ResNet v2 CNNs. Similar to [130], the authors in [148] use the deep CNN, called Decompose , Transfer, and Compose (DeTraC) and transfer learning approach for the classification of COVID-19 chest X-ray dataset (small). For evaluation the performance of $\mathrm{f}$ DeTraC, on transfer learning stage authors compared its performance with AlexNet, VGG19, ResNet, GoogleNet, and SqueezeNet. DeTraC with VGG19 has achieved the highest accuracy of $97.35 \%$, sensitivity of $98.23 \%$, and specificity of 96.34\%. DeTraC outperformed all pre-trained models with highest accuracies and has the ability to deal with data irregularity and the limited number of training images as well.

The authors in [149] proposed a two steps method for identifying the COVID-19 infected regions in the human lungs using the chest X-ray images. In the first step, Artificial Neural Network ANN and fractal methods are used for extracting the features of lung images. In the second step, CNN based segmentation methods are proposed to separate the infected tissue based on Lung MRI images. Result classification shows that the introduced CNN method has a higher exactness (93.2\%) and affectability (96.1\%) and is much better as compared to the deep neural network (DNN) strategy with a precision of $83.4 \%$ and affectability of $86 \%$.

Similar to [149], authors in [142] proposed two steps method to diagnosed the COVID-19 disease in patients. In the first step eight transfer learning techniques including, AlexNet, VGG16, VGG19, GoogleNet, ResNet18, ResNet50, ResNet101, InceptionV3, InceptionResNetV2, DenseNet201 and XceptionNet were used to extract the deep features from the X-ray images. The features ac- 
TABLE II: COVID-19 Diagnosing systems

\begin{tabular}{|c|c|c|c|c|c|c|}
\hline REF. & TECHNIQUE & DATASET & No. Of Images & Open Access & COVID Positive Cases & Dataset's URL \\
\hline [124] & Deep CNN & $\begin{array}{l}\text { COVIDx } \\
\text { (X-ray Images) }\end{array}$ & 13,975 & YES & 358 COVID CXR images & {$[125]$} \\
\hline [126] & ResNet50 & $\begin{array}{l}\text { COVIDx } \\
\text { X-ray Images }\end{array}$ & 5941 & YES & 68 COVID CXR images & [125] \\
\hline [33] & Deep CNN & $\begin{array}{l}3 \text { Datasets } \\
\text { (X-ray Images) }\end{array}$ & $\begin{array}{l}2800 \text { in DS1 } \\
11493 \text { in DS2 } \\
2772 \text { in DS3 }\end{array}$ & $\begin{array}{l}\text { YES } \\
\text { YES } \\
\text { YES }\end{array}$ & $\begin{array}{l}341 \text { COVID CXR images } \\
341 \text { COVID CXR images } \\
341 \text { COVID CXR images }\end{array}$ & $\begin{array}{l}{[127]} \\
{[128]} \\
{[129]}\end{array}$ \\
\hline [130] & $\begin{array}{l}\text { VGG19, MobileNet v2 } \\
\text { Inception, Xception } \\
\text { Inception ResNet v2 }\end{array}$ & $\begin{array}{l}2 \text { Datasets } \\
\text { (X-ray Images) }\end{array}$ & $\begin{array}{l}1427 \text { in DS1 } \\
1442 \text { in DS2 }\end{array}$ & $\begin{array}{l}\text { YES } \\
\text { YES }\end{array}$ & $\begin{array}{l}224 \text { COVID CXR images } \\
224 \text { COVID CXR images }\end{array}$ & {$[131][132]$} \\
\hline [133] & DeTraC & $\begin{array}{l}2 \text { Datasets } \\
\text { (Xray Images) }\end{array}$ & 196 & YES & 105 COVID CXR & [134] [127] \\
\hline [135] & VG-16 & Chest X-rays & 6523 & YES & 250 COVID CXR images & $\begin{array}{l}{[127][128]} \\
{[136][131]}\end{array}$ \\
\hline [137] & $\begin{array}{l}\text { SqueezeNet,MobileNetv2 } \\
\text { ResNet18, CheXNet } \\
\text { DenseNet201, InceptionV3 } \\
\text { ResNet101 }\end{array}$ & X-Ray images & 1,579 & YES & $\begin{array}{l}423 \text { COVID-19 } \\
1485 \text { viral pneumonia } \\
1579 \text { normal }\end{array}$ & $\begin{array}{l}{[138][139][131]} \\
{[140][141][128]}\end{array}$ \\
\hline [142] & $\begin{array}{l}\text { ResNet50, AlexNet } \\
\text { VGG16, VGG19, GoogleNet } \\
\text { ResNet18, ResNet50, ResNet101 } \\
\text { InceptionV3, InceptionResNetV2 } \\
\text { DenseNet201, XceptionNet } \\
\text { SVM }\end{array}$ & $\begin{array}{l}2 \text { Datasets } \\
\text { (X-ray Images) }\end{array}$ & $\begin{array}{l}682 \text { in Dsataset1 } \\
100 \text { in Dataset2 }\end{array}$ & $\begin{array}{l}\text { YES } \\
\text { YES }\end{array}$ & $\begin{array}{l}341 \text { COVID CXR images } \\
341 \text { COVID CXR images } \\
341 \text { COVID CXR images }\end{array}$ & [143] \\
\hline [144] & Truncated Inception Net & Six sub-databases & 3487 & YES & 423 COVID CXR images & $\begin{array}{l}{[138][139][131]} \\
{[140][141][128]}\end{array}$ \\
\hline [32] & Deep CNN & $\begin{array}{l}\text { Chest Xrays } \\
\text { CT images }\end{array}$ & $\begin{array}{l}170 \text { Xray } \\
360 \mathrm{CT}\end{array}$ & $\begin{array}{l}\text { YES } \\
\text { YES }\end{array}$ & $\begin{array}{l}85 \text { COVID CXR images } \\
203 \text { COVID CT images }\end{array}$ & $\begin{array}{l}{[145][131]} \\
{[146]}\end{array}$ \\
\hline
\end{tabular}

quired from these deep models are classified by SVM. To choose the best classification model, statistical analysis is carried out. The analysis shows that classification models ResNet50 plus SVM are statistically superior as compared to the other eight models. The proposed classification model for detection of COVID-19 has achieved $95.38 \%$ of accuracy. This model was trained on two datasets comprising of 682 and 100 X-ray images respectively. The experimental results showed the capability of DeTraC in the detection of COVID-19 cases from a comprehensive image data set collected from several hospitals around the world. High accuracy of $95.12 \%$ (with a sensitivity of $97.91 \%$, and a specificity of $91.87 \%$ ) was achieved by DeTraC in the detection of COVID-19 X-ray images from normal, and severe acute respiratory syndrome cases.

The authors in [137] created a dataset of X-ray images by combining publicly available datasets and images collected from recently published papers. The motive is to increase the size of database that trains the deep learning al- gorithms and develop highly accurate diagnosing system. The new dataset comprising of 423 COVID-19, 1485 viral pneumonia, and 1579 normal chest X-ray images. The authors trained eight CNN based algorithms including SqueezeNet [97], MobileNetv2, ResNet18 [150], InceptionV3, ResNet101 [151], CheXNet [152], DenseNet201 [153] [154], and VGG19 [155] using the new chest Xray database to classify and isolate the normal patients from COVID-19 patients. The simulation result showed that CheXNet outperforms the others. However, when a deeper version of DenseNet, when trained on a large augmented dataset is utilized, Dense201 outperforms CheXNet.

In [144], the authors proposed a deep learning-based CNN model, named Truncated Inception Net, to identify COVID-19 positive patients utilizing chest X-ray images. The proposed deep learning model is a modified version of the Inception Net V3 architecture [156]. In this research, experimental tests were done on six distinctive 
experimental datasets by combining Coronavirus positive, Pneumonia positive, Tuberculosis positive, and healthy chest X-rays. The proposed model outperforms other results in detecting COVID-19 cases from non-COVID ones. Furthermore, considering the number of parameters utilized in the proposed Truncated Inception Net model, authors claim that it is computationally efficient when compared to original Inception Net V3 model and other works proposed in the literature including, ResNet50, SVM, and COVID-Net.

In this research [32], the authors presented a basic, yet, powerful deep learning CNN model along with AlexNet, a modified transfer learning techniqu is proposed. AlexNet, a modified transfer learning for the detection of COVID-19 disease from chest X-ray and CT images. The proposed simple CNN architecture constitutes of only one convolutional layer that further consists of 16 filters followed by batch normalization, rectified linear unit (ReLU), two fully connected layers, SoftMax and a classification layer. To train the proposed approach, dataset is build through gathering the images (170 X-ray and $361 \mathrm{CT}$-scan images) from five different sources. The authors were able to accomplish $100 \%$ sensitivity when $\mathrm{X}$-ray is utilized to test the suspected patients for COVID19 and $90 \%$ sensitivity accomplished when CT images are utilized. Pretrained AlexNet,on the other hand, is not performing badly on X-ray images whereby it effectively identifies all COVID-19 images and effectively distinguishes $96 \%$ of the normal X-ray images. However, this isn't the case when pretrained AlexNet is used to separate COVID-19 CT images from their normal counterparts such that it only identifies $72 \%$ of the COVID-19 CT images accurately.

In the [135], the authors considered the time windows currently needed to acquire a COVID-19 diagnosis. In this paper, they propose an approach expected to drastically decrease this time window to around to 2.5 seconds. They propose and evaluate a deep learning technique that is based on transfer learning by exploiting the VGG-16 model. They built two models, the first was aimed to discern whether a chest X-ray is marked with a generic pulmonary disease. In the event that the X-ray is marked with generic pulmonary disease, they input the $\mathrm{X}$-beam to a subsequent model, expected to distinguish whether the pulmonary disease is COVID-19. The trial results which were obtained considering two different data sets for an aggregate of 6,523 chest X-beams, show a accuracy of 0.96 for the discrimination between healthy and generic pulmonary disease patients, and an exactness of 0.98 for the COVID-19 detection.

The authors in [137] collected X-ray images of 1579 normal, 1485 viral pneumonia and 423 COVID-19 positive pneumonia and, built a publicly available dataset. To asses the validity and a robustness of two different classification schemes, 8 distinct pre-trained deep learning models including MobileNetv2, SqueezeNet [157], ResNet18 [158], ResNet101, DenseNet201, CheXNet, Inceptionv3 and VGG19 were trained using this dataset.
One classification model was trained to classify COVID19 and normal X-ray images while second classification scheme was trained to classify normal, viral pneumonia and COVID-19 pneumonia images. The classification accuracy, precision, sensitivity, and specificity for both the schemes were $99.7 \%, 99.7 \%, 99.7 \%$ and $99.55 \%$ and $97.9 \%, 97.95 \%, 97.9 \%$, and $98.8 \%$, respectively.

\section{Lesson Learned and Challenges}

In this section, we discussed various machine learning algorithms that can be used for the diagnosis of COVID19 disease utilizing chest X-ray and CT-Scan. A variety of machine learning tools have been suggested by scientists for the diagnosis of COVID-19 through the efficient analysis of X-ray images; for instance, deep CNN architecture and its variants. The deep CNN architecture can successfully assist physicians and radiologists in the diagnosis of COVID-19. Based on the results of intelligent COVID19 diagnosing system, the physician can carry out the necessary examinations to confirm the case. This allows the confirmed cases to be isolated and given appropriate health care. However, at present, deep CNN in health care systems are still in their primary stages. There are many challenges towards the utilization of deep learning in combating COVID-19.

For instance, deep learning algorithms require a generous amount of training datasets for reliable and accurate results. The X-ray and CT-scan images are the primary diagnosing tools for COVID-19 disease and yet the number of available X-Ray images and CT-Scans are minimal. There is a need to add more images to the repositories. In this regard, collaboration with hospitals and health officials is required. This is because it allows collection of correct X-ray images of COVID-19 positive cases on a daily bases and and they can upload them to centralized repositories. However, there is general security and privacy concern of individuals about their health related information. As we described earlier, a patient may not agree to share her private health data due to the fear of loss or hacking of data. At present, COVID-19 has spread exponentially all around the globe. Specifically, after the emerging of Delta Plus variant which has become the most predominant variant. It poses a greater threat for those who have not been inoculated. According to one study [159], individuals with the Delta variant were more likely to be hospitalized than patients infected with Alpha or the original virus strains. Therefore, surviving in the era of COVID-19 is a higher priority than issues of privacy and security concerns. Individuals should be encourage to donate their COVID-19 related health information for the betterment of the planet and to save lives around the world. Another restriction of the present structure of deep learning is its reliance on human information. Human skill is principal to control the execution of deep learning methods and to efficiently combat the pandemic. 


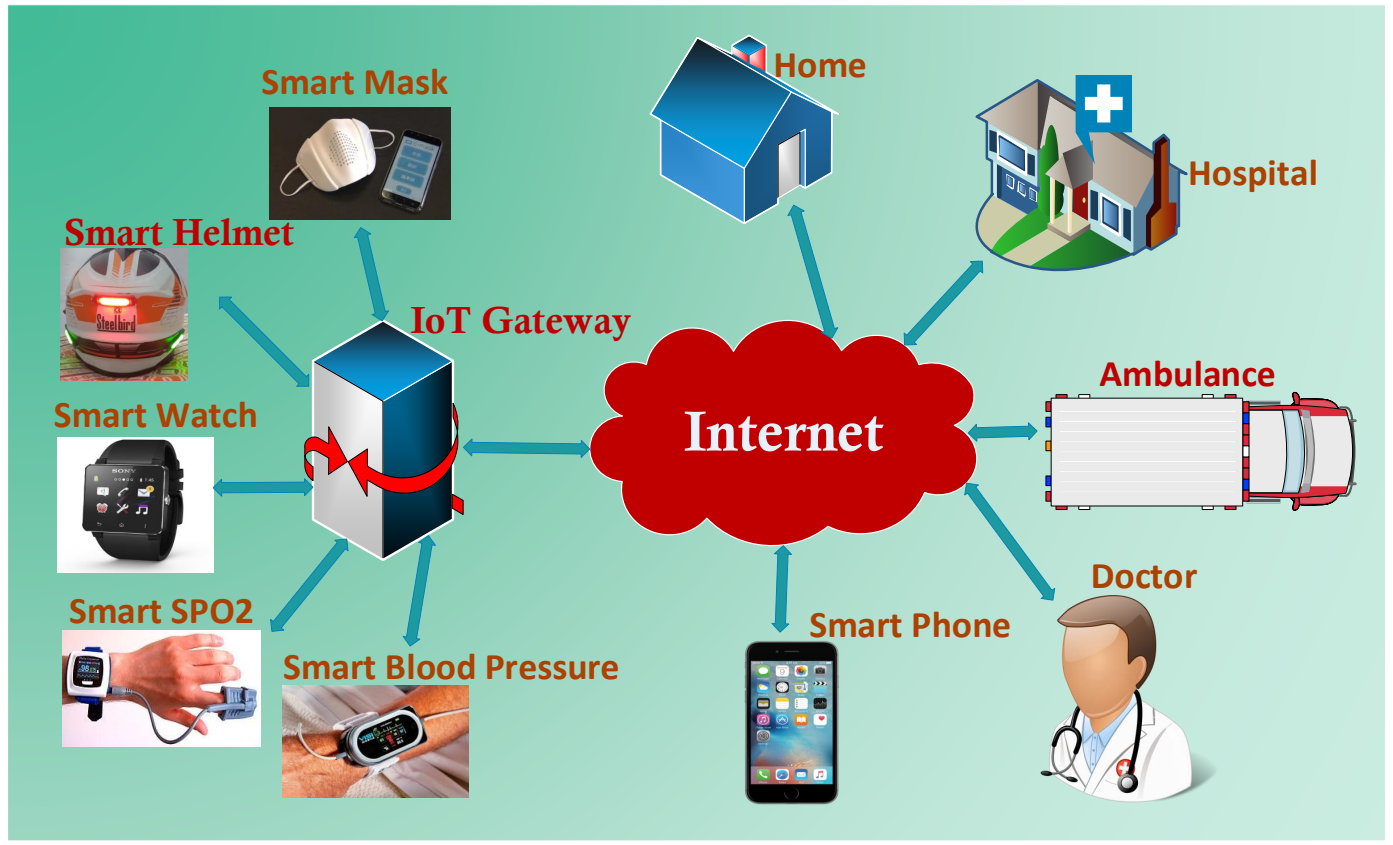

Fig. 4: Basic IoT-Healthcare Architecture.

\section{COVID-19 MONITORING SySTEMS}

In this section, we first present background in IoTs technology. Then, several research efforts towards developing IoT-based COVID-19 monitoring and diagnosis healthcare system are discussed.

\section{A. Background in IoTs Healthcare System}

An IoT is a network of devices which interact with each other through the machine to machine (M2M) correspondences [160] [161] [162], permitting data collection and exchange. The Harvard Business Review article [163], characterizes the IoT devices as "smart, connected devices". A device is called smart if it is able to gather information from its surroundings, analyze it using machine learning algorithms, and perform automated actions based on the outcomes without the human intervention. These smart devices are connected to IoT gateway (preferably to a smart fone via Bluetooth) and then the Internet and other technologies for different purposes (Fig. 4). For example, for smart patient tracking [164] [165] [166], to alert an hospital or healthcare authorities when a patient with a heart attack or injury is detected [167] [168] [44]. It can also be used to store detailed health information gathered by wearable sensor nodes such as a smart watch. Then the stored information on the private cloud or edge server can be downloaded by a patient's physician during a routine exam [169]. A typical smart healthcare IoT system comprises of the following components:

1) Sensor Nodes: Sensors are the key components of the smart healthcare IoT technology. Sensors collect data and information from surroundings and transfer it over a network to a dedicated central node or server or cloud for the analysis and taking the necessary actions [170]. The authors in [171] [172] [173] examine the distinctive existing wearable monitoring devices (respiration rate, heart rate, temperature, and oxygen saturation) and respiratory support systems (ventilators, CPAP devices, and oxygen therapy) which are often used to assist corona virus infected individuals. The devices are described based on the services they provide, their working methods as well as the comparative analysis of their pros and cons with cost. It is envisaged that wearable technology is only capable of providing initial indications that can reduce the spread of this pandemic. Sensor nodes are further classified as wearable sensors and vision based sensors:

(a) Wearable Sensors: The key to combating COVID-19 is to diagnose it earlier to prevent its wide spread across the globe. Wearable sensors can be used to help monitor and predict whether or not people are infected with the virus, based on signs such as body temperature, coughing patterns, and blood oxygen levels. With this information, the patient can assess his or her health situation and afterward choose to make a medical appointment before any other symptoms appear. Furthermore, the healthcare systems equipped with wearable sensors may assist physicians or surgeons to monitor infected patients or probable, and predict their future symptoms and health risks.

Wearable sensors can assist in monitoring other numerous health conditions including heart rate and pulse rate [174], blood pressure [175], calorie intake and burnt [176], smart exercises tracking, stress and anxiety tracking [177], menstrual tracking [178], and pregnancy tracking [179] [180] [181]. Commercially several smart watches, wrist bands, and chest traps are available for tracking an individuals pulse rate, heart rate etc. These include HRM-Tri by Garmin for monitoring 
heart rate [182], $\mathrm{H} 7$ by Polar (heart rate sensor) [183], FitBit PurePulse [184], and TomTom Spark Cardio [185].

(b) Vision Based Sensors (Cameras): They have been installed in physical locations such as around the home, hospitals, isolation wards, and ICUs etc., for continuously monitoring the patients suffering from critical health conditions such as Parkinson's Disease [186]. The authors in [187] suggest that wearable sensors in combination with cameras can help in monitoring the progression of Parkinson's Disease. Furthermore, the authors suggest that machine learning could lead to enhanced treatment plans in the future. This system could easily be adapted for other or additional diseases by modifying which wearable sensors are utilized. Machine learning algorithms could be implemented to vision cameras to identify the patient's progress, predict when they will be fully rehabilitated, and determine whether any medications or exercises are working better than others.

Utilization of these vision based devices may help in following a patient with Corona virus and provide care for recovering patients at home. The smart vision based system for detecting human body temperature are introduced in numerous nations where vision camera is integrated with the sensor and sends real-time data to the server. The framework likewise utilizes AI to recognize faces and matches it with the centralized database. For instance, the Department of Defense, US has used vision-cameras to capture the thermal images to identify people with high body temperatures [188]. In China, Baidu, one of the largest $\mathrm{AI}$ innovators and internet provider companies in the world, has developed computer vision powered infrared cameras to scan passengers' temperatures at Beijing's Qinghe Railway Station. These cameras can also recognize citizens who are not following the lockdown measures [189]. These smart camera vision systems provide significant advantages over hand-held thermometers because there is a safe distance between the operators and subjects and they require less manpower. A similar computer vision camera system has been deployed in Oxford, England, to monitor if the crowds are following the social distancing measures. An AI-based start-up in the USA - Landing AI, helmed by one of the most renowned AI experts in the world - Andrew $\mathrm{Ng}$, has also developed a vision based social distancing detection tool that monitors crowds and alerts the authorities whenever social distancing guidelines are breached [51].

2) IoT Gateway: An IoT Gateway is a physical or virtual device that connects sensors nodes and smart devices to cloud storage. It gives IoT devices an access to Internet. In simple words, it enables communication among smart devices and protocols and technologies. It collects massive data from a number of connected sensor nodes, processes it, and forwards to the cloud where machine learning algorithms and AI technology transform it into some meaningful and useful asset. To manage the IoT devices and sensor nodes it also receive data from the cloud. It means all the information going to IoT devices and cloud or vice versa must go through the connected IoT gateway.

3) A smart Fone App: A smart fone app works in association with the user's smart phone to collect proximity data using Bluetooth and to communicate with the server through the cellular data network. A smart phone APP is built to interact with users. First, the user has to create an account and answer general background. Then the smart phone app gathers the information through the sensor nodes and sends it to the edge node or server for training the data.

\section{B. IoT-Based Emerging Technologies for Monitoring and Diagnosing the COVID-19}

To maintain the physical distancing, monitoring health parameters, slowdown the COVID-19 spread, and enforce the precautionary measurements against the COVID-19, different frameworks and architectures, devices, and technologies have been proposed. In this section, we will provide a detail discussion and insights on these technologies.

1) IoT-Based Frameworks and Architectures: To monitor individuals health condition and notifies them to keep physical distancing amongst others, the authors in [190] introduced an IoT healthcare system, named "COVIDSAFE". The proposed framework includes a wearable sensor node by which the IoT sensor node can gather an individual's health parameters, for example, body temperature, heart rate, blood oxygen saturation ( SpO2) level, coughing pattern, a smartphone application that connects to the network to send the data to the edge server, a Bluetooth assistance based tracking system to alert about the safe distance zone violation, and a voice coughing detector that ceaselessly monitors the individuals voice and records the number of coughs and severity level of the cough. A subset of samples from the Khorshid COVID Cohort (KCC) [191] study was used to design the rules of the proposed decision-making system.

For early COVID-19 detection and to enforce the prevention, authors in [192] proposed a multi-layer (6layer) IoT architecture that connects smart cities, smart hospitals, smart transportation, smart cities and smart health care system etc. Several use cases and algorithms also proposed and discussed to prevent the COVID-19 spread such as use of smart ventilator and sensor nodes to monitor the oxygen level in confirmed COVID-19 cases, remote COVID-19 patients monitoring in hospitals, isolation wards and homes, smart sanitizing in homes, hospitals, and workplaces to disinfect the virus, smart grocery store for virus free shopping, smart gyms, smart nurseries and child care homes for children safety against the COVID-19 disease, to monitor the children' sleep pattern, breathing, body temperature, and use of AIassisted drones to measure six feet distance among people using distance measurement sensor nodes.

Authors in [193] propose a multilayer architecture comprising of sensors, actuator, communication system, cloud 


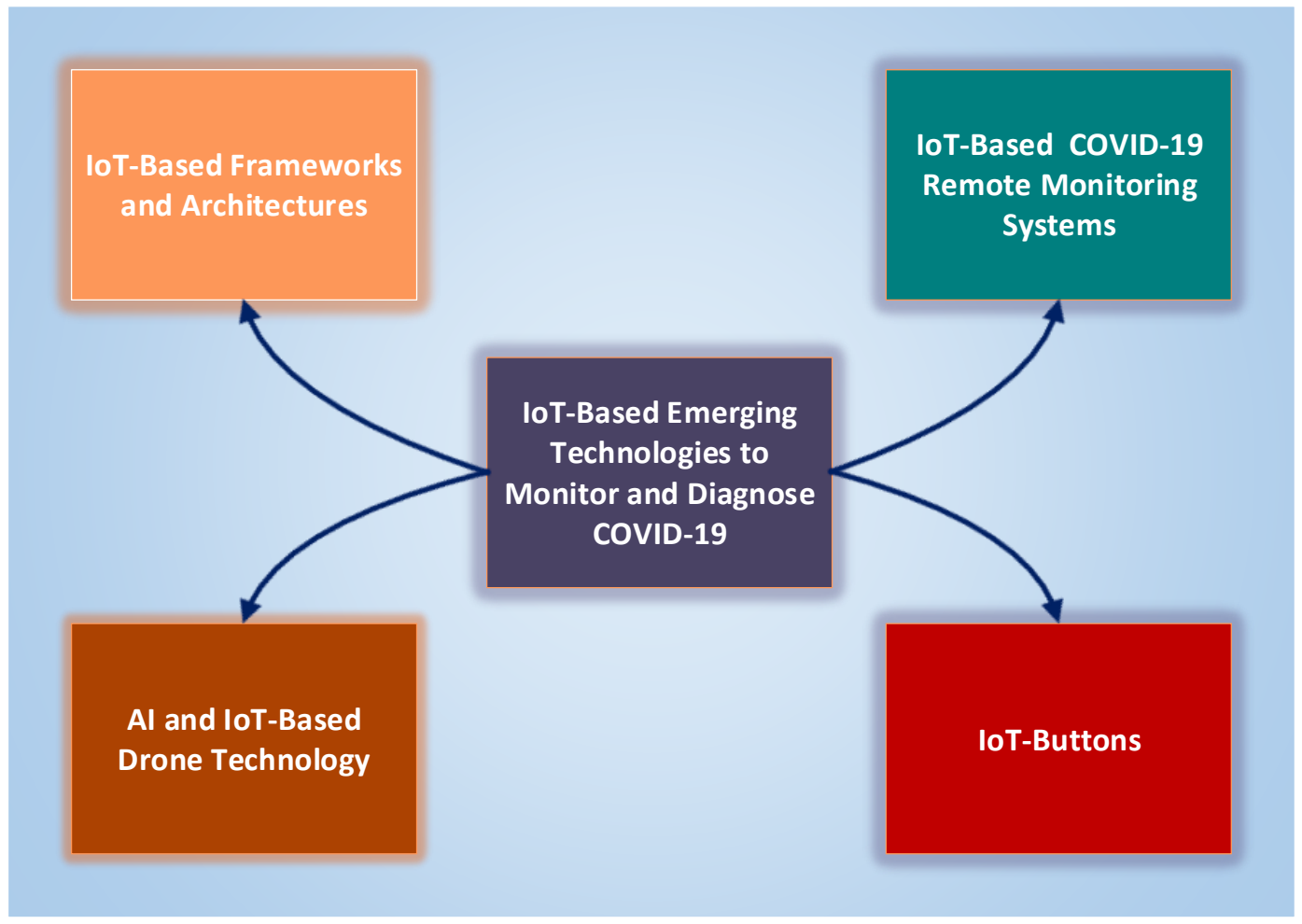

Fig. 5: IoT-Based Emerging Technologies for Monitoring and Diagnosing the COVID-19.

gateway and big date warehouse. Sensors collect the data from the physical world and transferred via the Internet. Actuators allow things to act or react according to the input received by the sensors. Data communication is through an gateway device that will further be transferred to the cloud gateway. In the big data warehouse, filtering of data, i.e., meaning full data, is extracted. A big data warehouse only contains structured data. Machine learning is utilized to make models of the systems based on requirements and received data.

The authors in [106] proposed a COVID-19 monitoring and detection system that utilized the wearable sensor nodes to collect the real time COVID-19 disease symptoms, travel history to suspicious areas, and contact history with potentially infected people. A dataset of 14251 confirmed COVID-19 cases from the COVID19 Open Research Dataset (CORD-19) repository [194] was used to train and built the predictive model using the eight ML algorithms including, Support Vector Machine, Neural Network, Naïve Bayes, K-Nearest Neighbor (KNN), Decision Table, Decision Stump, OneR, and ZeroR. The outcomes demonstrated that every one of these calculations, aside from the Decision Stump, OneR, and ZeroR accomplished an accuracy of more than $90 \%$. Utilizing the five best algorithms would give effective and accurate identification of potential cases of COVID-19. The authors in [195] introduce a new IoT-based architecture for contact and disease tracing, which specifically incorporates symptom-based detection that has been ignored in the prior art on tracing models. The ability of this framework to meaningfully merge real- time symptom information (from IoT sensor nodes) and confirmed Corona virus cases (from medical tests) gives a quick and proficient method of tracking the disease spread, which is ultimately useful for the scarce resources (e.g., Coronavirus test kits). The general framework is made out of four unique stages: $\mathrm{T} 1$ represents the initial stage of the system. At T2, every individual is checked for: 1. Symptoms, like fever, cough, and fatigue, with their corresponding infection rates 2 . Individuals with confirmed infections At this stage, individuals showing high symptoms are identified. At stage T3, individuals exposed to the infected patients or individuals showing high symptoms are identified depending on their proximity. Finally, in stage T4 the infected patients are quarantined, and the exposed individuals are isolated.

2) AI and IoT-assisted Drone Technology: Drones can and have offered many benefits during the global COVID19 pandemic. For instance, drones may play an important role during a quarantine to decrease the number of COVID-19 cases by lowering the interaction of healthcare workers with patients and contaminated areas. They can be very useful in keeping medical staff or non-infected people away from isolated patients. They can also be used to reach inaccessible or contaminated areas such as hospitals, laboratories, and isolated wards. Several research efforts in industry and academia have proposed the use of drone technology to monitor, detect, and diagnose the Corona virus and prevent or slowdown its spread rate.

Several developed countries including China, India, USA, Australia, and Spain etc., have successfully used drone 
technology to fight the COVID-19. For instance, a joint research project [196], worth budget of up to $\$ 1.5 \mathrm{M}$, by the Australian Department of Defense and University of South Australia is under the development process. The aim of the project to use the drone technology on vulnerable and potential risky areas such as old age homes and crowded areas, for monitoring and detection of infectious and respiratory conditions including body temperatures, heart rate, and respiratory rate. Likewise, Antwork Robotics, a Chinese drone delivery company owned by Terra Drone, is utilizing the drone technology for delivering medical supplies in COVID-19 infected areas in China [197]. The United States is also taking important steps to implement the drone technology to deliver the Medical equipment and medicines to the COVID-19 hotspot zones [198]. Another drone company "XAG" has also established a 5-million-yuan $(\$ 715,000)$ volunteer fund to support the use of their agriculture drones for spraying disinfectant to stop the spread of the corona virus. The Spanish military has recently adopted the use of agricultural drones made by "DJI", a leading Chinese drone manufacturer, to spray disinfecting chemicals over public spaces [199]. As an extraordinary measurement against the Corona virus, drone technology has been used to instruct the people without masks to use the mask in Chine. Likewise, the authorities in New Delhi (India) have used drone technology equipped with a thermal camera and vision camera for screening individuals and monitoring the crowd [200]. Similarly, drones outfitted with thermal cameras are being used to monitor body temperatures, allowing medical staff to identify new potential cases without having to touch those who might be infected [197]. Drones can prove to be highly useful for broadcasting important information in urban areas or regions that lack communication channels. The police authority in Madrid, Spain, used a drone equipped with a loudspeaker to advise people of the guidelines put in place regarding the state of emergency [201]. The corona virus crisis has also served to propel the use of drones to deliver food. The Chinese ecommerce company JD.com has launched its group of drones to carry out several food delivery tests that replace one hour transportation times with a flight of approximately 10 minutes [201].

Tracking people's geolocation can be another useful feature of drone technolgy. During the outbreak of a contagious disease, tracking the distance between people can provide valuable information. This information can be used to determine who the person has been in close contact with. The authors in [202], proposed multi-layer Drone-based COVID-19 monitoring and detection system comprising of thermal imaging system for measuring the social distancing, wearable sensor system for detecting the movement and collection of COVID-19 symptoms, edge computing system for analyzing the data uploaded by the Drones and sensor nodes, making the real time decisions, privacy and security system to ensure the confidentiality and privacy of individuals data. Algorithms for multiple use cases including remote monitoring, social distancing, and smart cenetization are proposed and implemented using the real time simulations. In the simulation, the proposed approach is tested for indoor and outdoor activities. Results show that a distance of 1200 kilometers can be covered in 2293 to 18900 min with a variation of 3-30 drones.

3) IOT-Based COVID-19 Remote Monitoring Systems: Remote Health monitoring means to monitor people's health outside the clinical settings. During the COVID-19 outbreak, remote monitoring has emerged as a new and powerful tool for the health officials, government health organizations and public. Remote monitoring can be very beneficial in managing and controlling the COVID-19 pandemic. For instance, remote monitoring system empowers observing patients from their homes that save governmental expense and time through measuring the changes in the patient in quarantine for medical readings. It can also be used in particular to gather pandemic data and obtain real-time clinical feedback. Since the start of COVID-19, researchers have proposed remote monitoring architectures, remote respiratory rate monitoring systems, remote continuous body temperature monitoring systems and remote heart rate monitoring systems. In the following section we provide insights to those systems.

COVID-19 Remote Monitoring Architectures: In a pandemic, wearable sensors equipped with AI technologies can be very useful in remote monitoring [175]. For example, if $\mathrm{SpO} 2$ level of the patient is less than $85 \%$, hospital practitioners will receive alerts through data-driven application and then send extra care services to the patient. Patients can also receive build corrective recommendations regarding medicines and extra precautions to be taken at home through IoT applications. The patient can also receive alerts based on threshold values through applications, especially when his/her health condition is critical. The authors in [203] propose a remote monitoring system that monitors the patients infected by the Corona virus, remotely and helps in protecting the lives of the health services member (like physicians and nurses) from infection. This smart system observes individuals with this disease based on putting numerous sensors to record various features of their patients every second. These parameters include measuring the patient's temperature, respiratory rate, heart rate, blood pressure, and time. It targets two types of individuals' the most serious medical conditions and disease and the least serious medical conditions in their house. The authors in [203] propose a framework named "E-Quarantine" that monitors the critically ill patients and predicts the emergency cases around 24 hours by $98.7 \%$ based on the supervised previous data such as blood PH level, heart rate, blood pressure, body temperature, and respiratory rate. The authors in [204] utilize the Fuzzy Inference System (FIS), which 
smartly monitors and predicts if an individual is the victim of Coronavirus or not. The proposed system comprises of two cloud-associated stages: the first is a training stage and the second is a validation stage. The training stage involves three levels: sensory layer, preprocessing layer and application layer. FIS is utilized in the prediction layer to predict the output. If sensory layer input parameters are appropriate, it will move through fuzzi fication into the fuzzy crisp inputs. The crisp set of input data is obtained and transformed into a fuzzy set utilizing fuzzy linguistic variables, fuzzy semantic terms and membership functions. The fluffy technologies proceed through the FIS into the next stage.

Remote Respiratory Rate Monitoring Systems: According to the world health organization, respiratory rate $>30 \mathrm{breath} / \mathrm{min}$ is a critical sign for the diagnosis of severe Corona virus infection [205]. At triage level, different values of respiratory rate are used by the health officials to make decisions on the use of supplemental oxygen. These values also assists in early detection of COVID-19 infection and assists in categorizing the patients according to the severity of the infection (mild/moderate/severe). These values are also used as the criterion for the assignment of patients to ICU or Isolation wards [206]. COVID-19 Patients requires continuous vital sign monitoring, even during everydaylife activities. Remote Respiratory Rate Monitoring will help health officials with the timely recognition of COVID-19 patient deterioration, thus contributing to the implementation of early intervention strategies [207]. There are many existing technological solutions such as sensors for the accurate monitoring of respiratory rate which if combined with the IoTs and AI can be used for the remote respiratory rate monitoring of COVID-19 patients [].The authors in [208] propose respiratory rate estimation using nasal breath sound recordings from a smartphone. Specifically, the proposed method detects nasal airflow using a built-in smartphone microphone or a headset microphone placed underneath the nose. These sound waves or signals can be converted into radio signals and transferred via high speed 5G technology to edge server or cloud storage for further analysis and making decisions. Patients that need continuous vital sign monitoring, even during everyday-life activities, can be equipped with wearable devices like smart garments (e.g., t-shirts or chest bands embedding sensors) (Massaroni et al., 2019b). Unlike most of the aforementioned technologies, smart garments may provide accurate and robust RR values even during daily activities [209]. The authors in [210] study was to assess the performance of a multi-sensor smart garment during walking and running activities using custom algorithms. In this study, we investigated the performances of a multisensors smart garment for the continuous and unobtrusive respiratory monitoring during physical activities (i.e., walking and running). Bespoke algorithms have been developed to optimize the system performances.
Remote Continuous Body Temperature Monitoring Systems: Many researchers have already proposed wearable devices for continuous body temperature monitoring which is also an important symptom used for the diagnosing of COVID-19 patients. These smart body temperature monitoring devices can take advantages of emerging technologies and can be used for remote monitoring of COVID-19 patients. For instance, based on several artificial neural networks, the authors in [211] proposed a wearable system which monitors the body temperature with a very high accuracy and efficiency. The authors in [212] presented a contactless continuous body temperature monitoring method in which they utilize a single thermal camera and deep-learning based face detection techniques to detect the forehead temperature of the individuals. The experimental results show that the overall mean absolute error (MAE) and root-meansquared-error (RMSE) of our proposed framework compared with industrial instrument are $0.375{ }^{\circ} \mathrm{C}$ and 0.439 ${ }^{\circ} \mathrm{C}$, respectively. The authors in [213] developed a small and comfortable wearable sensor device to continuously monitors the body temperature of a baby. The body temperature readings are transferred to the parents through a wireless communication system for the purpose of remote monitoring via the mobile phone. Another IoT based gadget known as Health Companion utilizing wearable computing was proposed in [214] which monitors the temperature and heartbeat on regular basis. This device aims to collect different parameters of the human body, helps individuals to monitor their health, and works with specialists to closely examine the patients' symptoms. The device warns the individual as well as the clinical staff if there is a significant increase in temperature or the individual has a fever.

4) IoT Buttons: IoT button is another technology that can be use in the crisis of COVID-19. IoT buttons are small, integrated, and Wi-Fi enabled devices that when pressed can send timely notifications or alerts to some central body for taking further actions or activate some pre-specified tasks.

A well-known example of IoT button is Amazon's "Dash" buttons that was firstly designed and developed for the amazon customers to supply the depleted products at their location such as, to re-ordering the detergent when it is running low at the laundry [215]. AWS IoT (Amazon Web Services Internet of Things)is Amazon's new reprogrammable and Wi-Fi enables IoT button, that can be programmed to control the internet-connected devices and services [216]. According to the Amazon's announcement new AWS button can be used as a remote control for Netflix, a check-in/check-out device for customers at hotel, one click way to order favorite pizza for delivery, unlock or start cars, open garage doors, call a cab, police, or people, track the use of household chores, order medications or products, or control home appliances as if people were using a remote control.

For example, if $\mathrm{SpO} 2$ level of the patient is less than $85 \%$, 
hospital practitioners will receive alerts through datadriven application and then send extra care services to the patient. Patients can also receive build corrective recommendations regarding medicines and extra precautions to be taken at home through IoT applications. He/she can also add his/her family members or friends to share his/her PGHD with different levels of access control due to security and privacy concerns. In general, due to privacy concerns, a patient might not agree to share his/her data, and in such cases, health devices will send data on a gateway device (e.g., patient's phone). However, some alerts can be false positives, and to address such errors, dense sensor networks along with med- ical devices can help to reduce false positives. Furthermore, classification techniques such as Hidden Markov Model (HMM) [31] can be used to classify anomalies in PGHD. This model can be deployed on cloud or gateway to detect false alerts. In hospitals or homes, IoT buttons can be configured to carry out a wide range of actions. For instance, If the health condition of a confirmed COVID-19 case isolated at home or in ICU gets worse, by pressing the button, a healthcare provider will be alerted, or family members will be notified in case of an emergency. IoT buttons can be used to maintain high cleaning standards and limit the number of hospital-acquired infections (HAIs) [217]. They can be use to issue alerts to management when hand sanitizers deployed at hospital facility need refilling, sanitation certain area, maintenance issues, warning them of any sanitation or maintenance issue that may pose a risk to public safety.

\section{Lesson Learned and Challenges}

Based on the benefits and suitability of IoTs in healthcare industry, we have investigated a few exploration endeavors towards creating IoT based COVID-19 monitoring and diagnosis health care systems. We have discussed numerous research efforts towards developing IoT-based COVID-19 remote monitoring systems along the wearable sensor nodes, IoT buttons, drone technology, and many architectures and frameworks proposed to control and manage the COVID-19 outbreak.

In terms of sensor nodes, we found that, a lot of suitable options are available to monitor various COVID19 symptoms such as body temperature, respiration rate, heart rate, temperature, and oxygen saturation level. We also found that, the healthcare systems equipped with wearable sensors and vision-based sensors may assist physicians and individuals to monitor infected patients or probable, and predict their future symptoms, assess health risks, and forecast their future conditions. However, development of integrated sensor technology for diagnosing and predicting COVID-19 disease need substantial attention from the research community in terms of accuracy, availability, wearability, and cost. There are still no allin-one sensor devices available that can monitor and predict all COVID-19 symptoms. As such, further research efforts should be made towards improving the quality of these sensors until they are highly accurate, reliable, and comfortably wearable. Research interests need to be focus on developing respiration rate, oxygen saturation, continuous body temperature monitoring devices which are more wearable than placing them on chest walls (smart patches), under the nose or on fingers and which also produce hospital-grade accuracy equivalent results without compromising energy efficiency and wearability and deployable worldwide. Likewise, we also concluded that continuous body temperature monitoring devices are extremely useful diagnostic tools and should be used and promoted in the current pandemic. However, their accuracy is limited by the degree of contact with the human body. More they are contacted with skin, more accurate results are achievable. However such devices are uncomfortable for patients. The authors in [?] proposed to embed the temperature sensing devices in textile to achieve the higher level of accuracy and comfortability. Therefore, engineers can also focus on developing smart textiles for measuring body temperature until some alternative or electronic printed patches on some soft backing support is manufactured. Furthermore, despite the numerous advantages of wearable devices, machine learning algorithms have not been explored in this area and providing a significant research opportunities.

In terms of remote monitoring systems, we found that it can play an important role in the context of the COVID19 pandemic. It may facilitate healthcare assistance for self-isolated COVID-19 patients as well as for all patients that have restricted access to medical services. The improvement of remote patient monitoring would also favor the implementation of timely and cost-effective healthcare services and consequently will reduce the burden from the healthcare facilities. However, successful implementation of remote monitoring system would require support of high speed cellular networks to upload/download the health related data with ultra low latency to or from sensors to an external storage (such as cloud or edge server). In this regard, use of 5G technology can allow to develop responsive and dynamic remote monitoring system. $5 \mathrm{G}$ is the fifth generation of wireless communication technology and expected to provide better performance in terms of higher speed, lower latency, wider range, increased availability, and more reliability [218] [219]. The integration of remote monitoring system with $5 \mathrm{G}$ network technology has the potential to revolutionize the healthcare sector and also provide significant research opportunities to computer scientists and telecommunication industry.

\section{Conclusion}

The novel Corona virus (SARS-CoV-2) has infected millions and caused the deaths of thousands of people in various countries. Currently, five vaccines, two from China, two from the U.S., and one from the UK, are being utilized around the world and numerous vaccines are under the trail process. In order to reach herd immunity, around $70 \%$ of the population would need to be 
inoculated. Although vaccination campaigns are in full swing around the world, it may take several years to hinder the spread of SARS-CoV-2. This is due to the fact that new variants are emerging day by day. There are currently four notable variants: alpha, beta, gamma, and delta. As new variants emerge, immunity provided by vaccines is threatened. Therefore, besides vaccines, other methods and technologies need to be utilized in order to overcome this pandemic. There have been massive efforts in various research areas and a wide variety of tools, technologies and techniques have been explored and developed to combat the war against this pandemic. Interestingly, machine learning algorithms and internet of Things (IoTs) technology were one of the first areas to be used to combat Covid-19. Up till now, several realtime and intelligent COVID-19 forecasting, diagnosing, and monitoring systems have been proposed to tackle the COVID-19 pandemic. To assist the research community by imparting an overall comprehension of the continuous exploration and potential research areas in COVID-19, in this survey article, we provide a comprehensive review of machine learning algorithms and Internet of Things (IoTs) technology to develop COVID-19 forecasting, diagnosing, and monitoring systems. Based on our indepth literature review, we provided a taxonomy based on the intelligent COVID-19 forecasting, diagnosing, and monitoring systems. We reviewed the available literature extensively under the proposed taxonomy and have analyzed a number of machine learning algorithms and IoTs which can be used in predicting the spread of COVID-19 and in diagnosing and monitoring the infected individuals. Furthermore, we identified the challenges and also provided our vision about the future research on COVID-19.

\section{ACKNOWLEDGMENT}

\section{REFERENCES}

[1] F. Jiang, L. Deng, L. Zhang, Y. Cai, C. W. Cheung, and Z. Xia, "Review of the clinical characteristics of coronavirus disease 2019 (covid-19)," Journal of general internal medicine, pp. 1-5, 2020.

[2] T. P. Velavan and C. G. Meyer, "The covid-19 epidemic," Tropical medicine \& international health, vol. 25, no. 3, p. 278, 2020.

[3] A. S. Fauci, H. C. Lane, and R. R. Redfield, "Covid-19-navigating the uncharted," 2020.

[4] L. Mullen, C. Potter, L. O. Gostin, A. Cicero, and J. B. Nuzzo, "An analysis of international health regulations emergency committees and public health emergency of international concern designations," $B M J$ global health, vol. 5, no. 6, p. e002502, 2020.

[5] President trump just declared the coronavirus pandemic a national emergency. here's what that means. [Online]. Available: tatnews.com/ 2020/03/13/national-emergency-coronavirus/

[6] Who coronavirus disease (covid-19) dashboard. [Online]. Available: https://covid19.who.int/table

[7] Z. Parolin, C. Wimer et al., "Forecasting estimates of poverty during the covid-19 crisis," Poverty and Social Policy Brief. New York, NY: Center on Poverty and Social Policy at the Columbia School of Social Work, vol. 4, no. 6, 2020.

[8] The global economic outlook during the covid19 pandemic: A changed world [Online]. Available: https://www.worldbank.org/en/news/feature/2020/ 06/08/the-global-economic-outlook-during-the-covid-19-I pandemic-a-changed-world
[9] C. Van Oosterhout, N. Hall, H. Ly, and K. M. Tyler, "Covid-19 evolution during the pandemic-implications of new sars-cov-2 variants on disease control and public health policies," 2021.

[10] D. Duong, "What's important to know about the new covid-19 variants?" 2021.

[11] A new variant of covid-19 has emerged in england - here is what it could mean for the pandemic and vaccines. [Online]. Available: https://www.forbes.com/sites/roberthart/2020/ 12/15/a-new-strain-of-covid-19-has-emerged-in- Iprotectl@ normalcr। relaxenglandhere-is-what-it-could-mean-for-the-pandemic lprotectl @ normalcr|relax-and-vaccines/?sh=1ea1d90e70f6

[12] South africa covid-19 strain: What we know about the new variant. [Online]. Available: https://www.wsj.com/articles/ the-new-covid-19-strain-in-south-africa-what-we-know-11609971229

[13] A new covid variant has been discovered in brazil - here's what we know so far. [Online]. Available: https://www.cnbc.com/2021/ 01/15/brazil-a-new-covid-variant-has-been-discovered-heres-what-। we-know-so-far.html

[14] Japan has found a new covid variant. here's how it compares to virus strains in the uk, south africa. [Online]. Available: https://www.cnbc.com/2021/01/11/ japan-covid-variant-how-it-compares-to-strains-in-uk-south-africa. html

[15] New covid strain spreading across u.s.: What we know. [Online]. Available: https://nymag.com/intelligencer/article/ what-is-the-new-covid-19-strain-that-shut-down-england-u-k.html

[16] What is the 'indian variant' of covid-19 that is spreading in victoria? [Online]. Available: https://www.abc.net.au/news/2021-05-28/ indian-covid-variant-in-victoria-infectiousness-symptoms/100171986

[17] Chinese covid-19 vaccines can be upgraded for variants in two months. [Online]. Available: https://news.cgtn.com/news/2021-01-27/ Chinese-COVID-19-vaccines-can-be-upgraded-to-deal-with-new-variants html

[18] Fauci: Covid vaccines are less effective against new strains - but still worth taking. [Online]. Available: https://www.forbes.com/sites/joewalsh/2021/02/01/ fauci-covid-vaccines-are-less-effective-against-new-strains

[19] Uk coronavirus strain is doubling in the u.s. every 10 days, study finds. [Online]. Available: https://www.cnbc.com/2021/02/07/ uk-coronavirus-strain-doubling-in-the-us-every-10-days-study-finds . html

[20] Indian coronavirus variant in the uk seems to be more transmissibleead more. [Online]. Available: https://www.newscientist.com/article/ 2277153indiancoronavirusvariantintheukseemstobemoretransmissible/

[21] N. M. Okba, M. A. Müller, W. Li, C. Wang, C. H. GeurtsvanKessel, V. M. Corman, M. M. Lamers, R. S. Sikkema, E. de Bruin, F. D. Chandler et al., "Severe acute respiratory syndrome coronavirus 2specific antibody responses in coronavirus disease patients," Emerging infectious diseases, vol. 26, no. 7, pp. 1478-1488, 2020.

[22] S. Khan, R. Siddique, M. A. Shereen, A. Ali, J. Liu, Q. Bai, N. Bashir, and M. Xue, "Emergence of a novel coronavirus, severe acute respiratory syndrome coronavirus 2: biology and therapeutic options," Journal of clinical microbiology, vol. 58, no. 5, 2020.

[23] G. Neumann, T. Noda, and Y. Kawaoka, "Emergence and pandemic potential of swine-origin h1n1 influenza virus," Nature, vol. 459, no. 7249, pp. 931-939, 2009.

[24] G. Chowell, S. M. Bertozzi, M. A. Colchero, H. Lopez-Gatell, C. Alpuche-Aranda, M. Hernandez, and M. A. Miller, "Severe respiratory disease concurrent with the circulation of h1n1 influenza," New England journal of medicine, vol. 361, no. 7, pp. 674-679, 2009.

[25] Y. Itoh, K. Shinya, M. Kiso, T. Watanabe, Y. Sakoda, M. Hatta, Y. Muramoto, D. Tamura, Y. Sakai-Tagawa, T. Noda et al., "In vitro and in vivo characterization of new swine-origin h1n1 influenza viruses," Nature, vol. 460, no. 7258, pp. 1021-1025, 2009.

[26] W. H. Organization et al., "Middle east respiratory syndrome coronavirus (mers-cov)," 2019.

[27] R. J. de Groot, S. C. Baker, R. S. Baric, C. S. Brown, C. Drosten, L. Enjuanes, R. A. Fouchier, M. Galiano, A. E. Gorbalenya, Z. A. Memish et al., "Commentary: Middle east respiratory syndrome coronavirus (mers-cov): announcement of the coronavirus study group," Journal of virology, vol. 87, no. 14, pp. 7790-7792, 2013.

[28] N. Ramadan and H. Shaib, "Middle east respiratory syndrome coronavirus (mers-cov): A review," Germs, vol. 9, no. 1, p. 35, 2019.

[29] A. F. Dugas, M. Jalalpour, Y. Gel, S. Levin, F. Torcaso, T. Igusa, and R. E. Rothman, "Influenza forecasting with google flu trends," PloS one, vol. 8, no. 2, p. e56176, 2013 
[30] M. J. Paul, M. Dredze, and D. Broniatowski, "Twitter improves influenza forecasting," PLoS currents, vol. 6, 2014.

[31] Y.-H. Hsieh, "2015 middle east respiratory syndrome coronavirus (mers-cov) nosocomial outbreak in south korea: insights from modeling," PeerJ, vol. 3, p. e1505, 2015.

[32] H. S. Maghdid, A. T. Asaad, K. Z. Ghafoor, A. S. Sadiq, and M. K. Khan, "Diagnosing covid-19 pneumonia from $\mathrm{x}$-ray and ct images using deep learning and transfer learning algorithms," arXiv preprint arXiv:2004.00038, 2020.

[33] A. Narin, C. Kaya, and Z. Pamuk, "Automatic detection of coronavirus disease (covid-19) using $\mathrm{X}$-ray images and deep convolutional neural networks," arXiv preprint arXiv:2003.10849, 2020.

[34] T. Ai, Z. Yang, H. Hou, C. Zhan, C. Chen, W. Lv, Q. Tao, Z. Sun, and L. Xia, "Correlation of chest ct and rt-per testing in coronavirus disease 2019 (covid-19) in china: a report of 1014 cases," Radiology, p. 200642, 2020

[35] C. F. Pasluosta, H. Gassner, J. Winkler, J. Klucken, and B. M. Eskofier, "An emerging era in the management of parkinson's disease: wearable technologies and the internet of things," IEEE journal of biomedical and health informatics, vol. 19, no. 6, pp. 1873-1881, 2015.

[36] H. T. Cheng and W. Zhuang, "Bluetooth-enabled in-home patient monitoring system: Early detection of alzheimer's disease," IEEE Wireless Communications, vol. 17, no. 1, pp. 74-79, 2010

[37] S.-H. Chang, R.-D. Chiang, S.-J. Wu, and W.-T. Chang, "A contextaware, interactive $\mathrm{m}$-health system for diabetics," IT professional, vol. 18, no. 3, pp. 14-22, 2016

[38] D. Oletic and V. Bilas, "Energy-efficient respiratory sounds sensing for personal mobile asthma monitoring," Ieee sensors journal, vol. 16, no. 23 , pp. 8295-8303, 2016.

[39] M. Singh and N. Jain, "Performance and evaluation of smartphone based wireless blood pressure monitoring system using bluetooth," IEEE Sensors Journal, vol. 16, no. 23, pp. 8322-8328, 2016.

[40] Y. Zhang, M. Berthelot, and B. Lo, "Wireless wearable photoplethysmography sensors for continuous blood pressure monitoring," in 2016 IEEE Wireless Health (WH). IEEE, 2016, pp. 1-8.

[41] D. Griggs, M. Sharma, A. Naghibi, C. Wallin, V. Ho, K. Barbosa, T. Ghirmai, H. Cao, and S. K. Krishnan, "Design and development of continuous cuff-less blood pressure monitoring devices," in 2016 IEEE SENSORS. IEEE, 2016, pp. 1-3.

[42] Y.-L. Zheng, B. P. Yan, Y.-T. Zhang, and C. C. Poon, "An armband wearable device for overnight and cuff-less blood pressure measurement," IEEE transactions on biomedical engineering, vol. 61, no. 7, pp. 2179-2186, 2014.

[43] H. Lin, W. Xu, N. Guan, D. Ji, Y. Wei, and W. Yi, "Noninvasive and continuous blood pressure monitoring using wearable body sensor networks," IEEE intelligent systems, vol. 30, no. 6, pp. 38-48, 2015.

[44] G. Wolgast, C. Ehrenborg, A. Israelsson, J. Helander, E. Johansson, and H. Manefjord, "Wireless body area network for heart attack detection [education corner]," IEEE antennas and propagation magazine, vol. 58, no. 5, pp. 84-92, 2016.

[45] S. B. Baker, W. Xiang, and I. Atkinson, "Internet of things for smart healthcare: Technologies, challenges, and opportunities," IEEE Access, vol. 5, pp. 26 521-26 544, 2017.

[46] A. S. Ahuja, V. P. Reddy, and O. Marques, "Artificial intelligence and covid-19: A multidisciplinary approach," 2020.

[47] A. A. Hussain, O. Bouachir, F. Al-Turjman, and M. Aloqaily, "Ai techniques for covid-19," IEEE Access, vol. 8, pp. 128776-128795, 2020

[48] J. Bullock, K. H. Pham, C. S. N. Lam, M. Luengo-Oroz et al., "Mapping the landscape of artificial intelligence applications against covid-19," arXiv preprint arXiv:2003.11336, 2020.

[49] A. Shoeibi, M. Khodatars, R. Alizadehsani, N. Ghassemi, M. Jafari, P. Moridian, A. Khadem, D. Sadeghi, S. Hussain, A. Zare et al., "Automated detection and forecasting of covid-19 using deep learning techniques: A review," arXiv preprint arXiv:2007.10785, 2020.

[50] M. Kamal, A. Aljohani, and E. Alanazi, "Iot meets covid-19: Status, challenges, and opportunities," arXiv preprint arXiv:2007.12268, 2020.

[51] J. Shuja, E. Alanazi, W. Alasmary, and A. Alashaikh, "Covid-19 open source data sets: A comprehensive survey," Applied Intelligence, pp. 1-30, 2020.

[52] Covid-19 report: Publications, clinical trials, funding covid19.dimensions.ai. [Online]. Available: https://reports.dimensions.ai/ covid-19/

[53] F. S. Lu, M. W. Hattab, C. L. Clemente, M. Biggerstaff, and M. Santillana, "Improved state-level influenza nowcasting in the united states leveraging internet-based data and network approaches," Nature communications, vol. 10, no. 1, pp. 1-10, 2019.
[54] G. Briscese, N. Lacetera, M. Macis, and M. Tonin, "Compliance with covid-19 social-distancing measures in italy: the role of expectations and duration," National Bureau of Economic Research, Tech. Rep., 2020.

[55] J. A. Lewnard and N. C. Lo, "Scientific and ethical basis for socialdistancing interventions against covid-19," The Lancet Infectious Diseases, vol. 20, no. 6, pp. 631-633, 2020.

[56] J. Willan, A. J. King, K. Jeffery, and N. Bienz, "Challenges for nhs hospitals during covid-19 epidemic," 2020.

[57] S. Lu, X. Qiu, J. Shi, N. Li, Z.-H. Lu, P. Chen, M.-M. Yang, F.-Y. Liu, W.-J. Jia, and Y. Zhang, "A pathological brain detection system based on extreme learning machine optimized by bat algorithm," CNS \& Neurological Disorders-Drug Targets (Formerly Current Drug TargetsCNS \& Neurological Disorders), vol. 16, no. 1, pp. 23-29, 2017.

[58] Y.-D. Zhang, G. Zhao, J. Sun, X. Wu, Z.-H. Wang, H.-M. Liu, V. V. Govindaraj, T. Zhan, and J. Li, "Smart pathological brain detection by synthetic minority oversampling technique, extreme learning machine, and jaya algorithm," Multimedia Tools and Applications, vol. 77, no. 17, pp. 22 629-22 648, 2018.

[59] S. M. McKinney, M. Sieniek, V. Godbole, J. Godwin, N. Antropova, H. Ashrafian, T. Back, M. Chesus, G. C. Corrado, A. Darzi et al., "International evaluation of an ai system for breast cancer screening," Nature, vol. 577, no. 7788, pp. 89-94, 2020.

[60] M. D. Podolsky, A. A. Barchuk, V. I. Kuznetcov, N. F. Gusarova, V. S. Gaidukov, and S. A. Tarakanov, "Evaluation of machine learning algorithm utilization for lung cancer classification based on gene expression levels," Asian Pacific Journal of Cancer Prevention, vol. 17, no. 2, pp. 835-838, 2016

[61] S. L. Bergquist, G. A. Brooks, N. L. Keating, M. B. Landrum, and S. Rose, "Classifying lung cancer severity with ensemble machine learning in health care claims data," Proceedings of machine learning research, vol. 68 , p. $25,2017$.

[62] D. Jiang, J. Liao, H. Duan, Q. Wu, G. Owen, C. Shu, L. Chen, Y. He, Z. Wu, D. He et al., "A machine learning-based prognostic predictor for stage iii colon cancer," Scientific reports, vol. 10, no. 1, pp. 1-9, 2020.

[63] I. Pacal, D. Karaboga, A. Basturk, B. Akay, and U. Nalbantoglu, "A comprehensive review of deep learning in colon cancer," Computers in Biology and Medicine, p. 104003, 2020.

[64] L. Liu, S. Zhao, H. Chen, and A. Wang, "A new machine learning method for identifying alzheimer's disease," Simulation Modelling Practice and Theory, vol. 99, p. 102023, 2020.

[65] H. Yin, B. Mukadam, X. Dai, and N. Jha, "Diabdeep: Pervasive diabetes diagnosis based on wearable medical sensors and efficient neural networks," IEEE Transactions on Emerging Topics in Computing, 2019.

[66] P. K. Sahoo, S. K. Mohapatra, and S.-L. Wu, "Analyzing healthcare big data with prediction for future health condition," IEEE Access, vol. 4 pp. 9786-9799, 2016.

[67] R. K. Pathinarupothi, P. Durga, and E. S. Rangan, "Iot-based smart edge for global health: Remote monitoring with severity detection and alerts transmission," IEEE Internet of Things Journal, vol. 6, no. 2, pp. 2449-2462, 2018.

[68] F. Zantalis, G. Koulouras, S. Karabetsos, and D. Kandris, "A review of machine learning and iot in smart transportation," Future Internet, vol. 11, no. 4, p. 94, 2019.

[69] S. R. Sahoo and B. Gupta, "Popularity-based detection of malicious content in facebook using machine learning approach," in First International Conference on Sustainable Technologies for Computational Intelligence. Springer, 2020, pp. 163-176.

[70] G. Linden, B. Smith, and J. York, "Amazon. com recommendations: Item-to-item collaborative filtering," IEEE Internet computing, vol. 7 , no. 1, pp. 76-80, 2003.

[71] J. O. Awoyemi, A. O. Adetunmbi, and S. A. Oluwadare, "Credit card fraud detection using machine learning techniques: A comparative analysis," in 2017 International Conference on Computing Networking and Informatics (ICCNI). IEEE, 2017, pp. 1-9.

[72] M. Tektaş, "Weather forecasting using anfis and arima models," Environmental Research, Engineering and Management, vol. 51, no. 1, pp. $5-10,2010$.

[73] A. G. Salman, B. Kanigoro, and Y. Heryadi, "Weather forecasting using deep learning techniques," in 2015 international conference on advanced computer science and information systems (ICACSIS). Ieee, 2015, pp. 281-285.

[74] R. Lee and J. Liu, "ijade weatherman: a weather forecasting system using intelligent multiagent-based fuzzy neuro network," IEEE Transactions on Systems, Man, and Cybernetics, Part C (Applications and Reviews), vol. 34, no. 3, pp. 369-377, 2004. 
[75] R. Choudhry and K. Garg, "A hybrid machine learning system for stock market forecasting," World Academy of Science, Engineering and Technology, vol. 39, no. 3, pp. 315-318, 2008.

[76] M. R. Hassan and B. Nath, "Stock market forecasting using hidden markov model: a new approach," in 5th International Conference on Intelligent Systems Design and Applications (ISDA'05). IEEE, 2005, pp. 192-196.

[77] M. Fatima, M. Pasha et al., "Survey of machine learning algorithms for disease diagnostic," Journal of Intelligent Learning Systems and Applications, vol. 9, no. 01, p. 1, 2017.

[78] P. C. Sen, M. Hajra, and M. Ghosh, "Supervised classification algorithms in machine learning: A survey and review," in Emerging technology in modelling and graphics. Springer, 2020, pp. 99-111.

[79] M. Alloghani, D. Al-Jumeily, J. Mustafina, A. Hussain, and A. J. Aljaaf, "A systematic review on supervised and unsupervised machine learning algorithms for data science," Supervised and Unsupervised Learning for Data Science, pp. 3-21, 2020

[80] Z.-Q. Huang, Y.-C. Chen, and C.-Y. Wen, "Real-time weather monitoring and prediction using city buses and machine learning," Sensors, vol. 20 , no. 18 , p. $5173,2020$.

[81] S. Mullainathan and J. Spiess, "Machine learning: an applied econometric approach," Journal of Economic Perspectives, vol. 31, no. 2, pp. 87-106, 2017.

[82] A. Strehl and M. Littman, "Online linear regression and its application to model-based reinforcement learning," Advances in Neural Information Processing Systems, vol. 20, pp. 1417-1424, 2007.

[83] M. Khanum, T. Mahboob, W. Imtiaz, H. A. Ghafoor, and R. Sehar, "A survey on unsupervised machine learning algorithms for automation, classification and maintenance," International Journal of Computer Applications, vol. 119, no. 13, 2015.

[84] A. Likas, N. Vlassis, and J. J. Verbeek, "The global k-means clustering algorithm," Pattern recognition, vol. 36, no. 2, pp. 451-461, 2003.

[85] M. Sarkar and T.-Y. Leong, "Application of k-nearest neighbors algorithm on breast cancer diagnosis problem." in Proceedings of the AMIA Symposium. American Medical Informatics Association, 2000, p. 759.

[86] E. Schubert, J. Sander, M. Ester, H. P. Kriegel, and X. Xu, "Dbscan revisited, revisited: why and how you should (still) use dbscan," $A C M$ Transactions on Database Systems (TODS), vol. 42, no. 3, pp. 1-21, 2017.

[87] A. Singhal, P. Singh, B. Lall, and S. D. Joshi, "Modeling and prediction of covid-19 pandemic using gaussian mixture model," Chaos, Solitons \& Fractals, vol. 138, p. 110023, 2020.

[88] K. K. Sharma and A. Seal, "Multi-view spectral clustering for uncertain objects," Information Sciences, vol. 547, pp. 723-745, 2021.

[89] A. Sholokhov, M. Sahidullah, and T. Kinnunen, "Semi-supervised speech activity detection with an application to automatic speaker verification," Computer Speech \& Language, vol. 47, pp. 132-156, 2018

[90] S. Dumais and H. Chen, "Hierarchical classification of web content," in Proceedings of the 23rd annual international ACM SIGIR conference on Research and development in information retrieval, 2000, pp. 256263.

[91] J. Cao and L. Xiong, "Protein sequence classification with improved extreme learning machine algorithms," BioMed research international, vol. 2014, 2014

[92] V. G. P. Rathi and S. Palani, "Brain tumor detection and classification using deep learning classifier on mri images," Research Journal of Applied Sciences, Engineering and Technology, vol. 10, no. 2, pp. 177$187,2015$.

[93] S. Sajid, S. Hussain, and A. Sarwar, "Brain tumor detection and segmentation in mr images using deep learning," Arabian Journal for Science and Engineering, vol. 44, no. 11, pp. 9249-9261, 2019.

[94] L. Shen, L. R. Margolies, J. H. Rothstein, E. Fluder, R. McBride, and W. Sieh, "Deep learning to improve breast cancer detection on screening mammography," Scientific reports, vol. 9, no. 1, pp. 1-12, 2019.

[95] Y. LeCun, Y. Bengio et al., "Convolutional networks for images, speech, and time series," The handbook of brain theory and neural networks, vol. 3361, no. 10, p. 1995, 1995

[96] Q. Zhang, D. Zhou, and X. Zeng, "Heartid: A multiresolution convolutional neural network for ecg-based biometric human identification in smart health applications," Ieee Access, vol. 5, pp. 11 805-11 816, 2017.

[97] F. N. Iandola, S. Han, M. W. Moskewicz, K. Ashraf, W. J. Dally, and K. Keutzer, "Squeezenet: Alexnet-level accuracy with 50x fewer parameters and $<0.5 \mathrm{mb}$ model size," arXiv preprint arXiv:1602.07360, 2016.
[98] L. Wang, S. Guo, W. Huang, and Y. Qiao, "Places205-vggnet models for scene recognition," arXiv preprint arXiv:1508.01667, 2015.

[99] P. Ballester and R. Araujo, "On the performance of googlenet and alexnet applied to sketches," in Proceedings of the AAAI Conference on Artificial Intelligence, vol. 30, no. 1, 2016.

[100] Y. Li, "Deep reinforcement learning: An overview," arXiv preprint arXiv:1701.07274, 2017.

[101] M. Mahmud, M. S. Kaiser, A. Hussain, and S. Vassanelli, "Applications of deep learning and reinforcement learning to biological data," IEEE transactions on neural networks and learning systems, vol. 29, no. 6, pp. 2063-2079, 2018.

[102] D. Liu, L. Clemente, C. Poirier, X. Ding, M. Chinazzi, J. T. Davis, A. Vespignani, and M. Santillana, "A machine learning methodology for real-time forecasting of the 2019-2020 covid-19 outbreak using internet searches, news alerts, and estimates from mechanistic models," arXiv preprint arXiv:2004.04019, "2020".

[103] Z. Hu, Q. Ge, L. Jin, and M. Xiong, "Artificial intelligence forecasting of covid-19 in china," arXiv preprint arXiv:2002.07112, 2020.

[104] S. Sengupta, S. Mugde, and G. Sharma, "Covid-19 pandemic data analysis and forecasting using machine learning algorithms," medRxiv, 2020.

[105] R. Majhi, R. Thangeda, R. P. Sugasi, and N. Kumar, "Analysis and prediction of covid-19 trajectory: A machine learning approach," Journal of Public Affairs, p. e2537, 2020.

[106] M. Otoom, N. Otoum, M. A. Alzubaidi, Y. Etoom, and R. Banihani, "An iot-based framework for early identification and monitoring of covid-19 cases," Biomedical Signal Processing and Control, vol. 62, p. 102149,2020

[107] S. I. Alzahrani, I. A. Aljamaan, and E. A. Al-Fakih, "Forecasting the spread of the covid-19 pandemic in saudi arabia using arima prediction model under current public health interventions," Journal of infection and public health, vol. 13, no. 7, pp. 914-919, 2020.

[108] Z. Malki, E.-S. Atlam, A. E. Hassanien, G. Dagnew, M. A. Elhosseini, and I. Gad, "Association between weather data and covid-19 pandemic predicting mortality rate: Machine learning approaches," Chaos, Solitons \& Fractals, vol. 138, p. 110137, 2020.

[109] “Covid-19 dataset," https://www.kaggle.com/imdevskp/ corona-virus-report, accessed: 2020.

[110] "Covid19 global weatherdata," https://www.kaggle.com/ winterpierre91/covid19-global-weather-data, accessed: 2020.

[111] P. Kumar, H. Kalita, S. Patairiya, Y. D. Sharma, C. Nanda, M. Rani, J. Rahmani, and A. S. Bhagavathula, "Forecasting the dynamics of covid-19 pandemic in top 15 countries in april 2020: Arima model with machine learning approach," medRxiv, 2020.

[112] M. Alazab, A. Awajan, A. Mesleh, A. Abraham, V. Jatana, and S. Alhyari, "Covid-19 prediction and detection using deep learning," International Journal of Computer Information Systems and Industrial Management Applications, vol. 12, pp. 168-181, 2020

[113] "Covid-19 coronavirus pandemic," https://www.worldometers.info/ coronavirus/, accessed: 2021-06-06

[114] T. Chakraborty and I. Ghosh, "Real-time forecasts and risk assessment of novel coronavirus (covid-19) cases: A data-driven analysis," Chaos, Solitons \& Fractals, p. 109850, 2020

[115] İ. Kırbaş, A. Sözen, A. D. Tuncer, and F. Ş. Kazancıŏglu, "Comperative analysis and forecasting of covid-19 cases in various european countries with arima, narnn and 1stm approaches," Chaos, Solitons \& Fractals, p. 110015,2020 .

[116] C. Bretó, D. He, E. L. Ionides, A. A. King et al., "Time series analysis via mechanistic models," The Annals of Applied Statistics, vol. 3, no. 1, pp. 319-348, 2009.

[117] J.-F. Daoust, "Elderly people and responses to covid-19 in 27 countries," PloS one, vol. 15, no. 7, p. e0235590, 2020.

[118] J. Quilty and J. Adamowski, "Addressing the incorrect usage of wavelet-based hydrological and water resources forecasting models for real-world applications with best practices and a new forecasting framework," Journal of hydrology, vol. 563, pp. 336-353, 2018.

[119] J. Demongeot, Y. Flet-Berliac, and H. Seligmann, "Temperature decreases spread parameters of the new covid-19 case dynamics," Biology, vol. 9, no. 5, p. 94, 2020.

[120] O. Vandenberg, D. Martiny, O. Rochas, A. van Belkum, and Z. Kozlakidis, "Considerations for diagnostic covid-19 tests," Nature Reviews Microbiology, pp. 1-13, 2020.

[121] Different types of covid-19 tests explained. [Online]. Available: https://health.ucdavis.edu/health-news/newsroom/ different-types-of-covid-19-tests-explained/2020/11 
[122] G. C. Mak, P. K. Cheng, S. S. Lau, K. K. Wong, C. Lau, E. T. Lam, R. C. Chan, and D. N. Tsang, "Evaluation of rapid antigen test for detection of sars-cov-2 virus," Journal of Clinical Virology, vol. 129, p. 104500, 2020.

[123] A. Scohy, A. Anantharajah, M. Bodéus, B. Kabamba-Mukadi, A. Verroken, and H. Rodriguez-Villalobos, "Low performance of rapid antigen detection test as frontline testing for covid-19 diagnosis," Journal of Clinical Virology, vol. 129, p. 104455, 2020.

[124] L. Wang, Z. Q. Lin, and A. Wong, "Covid-net: A tailored deep convolutional neural network design for detection of covid-19 cases from chest X-ray images," Scientific Reports, vol. 10, no. 1, pp. 1-12, 2020 .

[125] "lindawangg/covid-net," https://github.com/lindawangg/COVID-Net, accessed: 2021-06-5.

[126] M. Farooq and A. Hafeez, "Covid-resnet: A deep learning framework for screening of covid19 from radiographs," arXiv preprint arXiv:2003.14395, 2020

[127] J. P. Cohen, P. Morrison, L. Dao, K. Roth, T. Q. Duong, and M. Ghassemi, "Covid-19 image data collection: Prospective predictions are the future," arXiv preprint arXiv:2006.11988, 2020.

[128] X. Wang, Y. Peng, L. Lu, Z. Lu, M. Bagheri, and R. M. Summers, "ChestX-ray8: Hospital-scale chest X-ray database and benchmarks on weakly-supervised classification and localization of common thorax diseases," in Proceedings of the IEEE conference on computer vision and pattern recognition, 2017, pp. 2097-2106.

[129] P. Mooney, "Chest x-ray images (pneumonia)," kaggle, Marzo, 2018.

[130] I. D. Apostolopoulos and T. A. Mpesiana, "Covid-19: automatic detection from x-ray images utilizing transfer learning with convolutional neural networks," Physical and Engineering Sciences in Medicine, vol. 43, no. 2, pp. 635-640, 2020.

[131] “ieee8023/covid-chestxray-dataset," https://github.com/ieee8023/ covid-chestxray-dataset, accessed: 2021-06-5.

[132] "Covid-19 x rays," https://www.kaggle.com/andrewmvd/ convid19-X-rays, accessed: 2021-06-5.

[133] A. Abbas, M. M. Abdelsamea, and M. M. Gaber, "Classification of covid-19 in chest $\mathrm{x}$-ray images using detrac deep convolutional neural network," Applied Intelligence, vol. 51, no. 2, pp. 854-864, 2021.

[134] S. Candemir, S. Jaeger, K. Palaniappan, J. P. Musco, R. K. Singh Z. Xue, A. Karargyris, S. Antani, G. Thoma, and C. J. McDonald "Lung segmentation in chest radiographs using anatomical atlases with nonrigid registration," IEEE transactions on medical imaging, vol. 33, no. 2, pp. 577-590, 2013

[135] L. Brunese, F. Mercaldo, A. Reginelli, and A. Santone, "Explainable deep learning for pulmonary disease and coronavirus covid-19 detection from x-rays," Computer Methods and Programs in Biomedicine, vol. 196, p. 105608, 2020

[136] T. Ozturk, M. Talo, E. A. Yildirim, U. B. Baloglu, O. Yildirim, and U. R. Acharya, "Automated detection of covid-19 cases using deep neural networks with x-ray images," Computers in biology and medicine, vol. 121, p. 103792, 2020.

[137] M. E. Chowdhury, T. Rahman, A. Khandakar, R. Mazhar, M. A. Kadir, Z. B. Mahbub, K. R. Islam, M. S. Khan, A. Iqbal, N. Al Emadi et al., "Can ai help in screening viral and covid-19 pneumonia?" IEEE Access, vol. 8, pp. 132665-132676, 2020.

[138] "Covid-19 radiography database," https://www.kaggle.com/ tawsifurrahman/covid19-radiography-database, accessed: 2021-06-5.

[139] "Covid-19 database," https://sirm.org/category/senza-categoria/ covid-19/\#, accessed: 2021-06-5.

[140] "Radiopedia," https://radiopaedia.org, accessed: 2021-06-5.

[141] "Chest imaging," https://threadreaderapp.com/thread/ 1243928581983670272.html, accessed: 2021-06-5.

[142] P. K. Sethy, S. K. Behera, P. K. Ratha, and P. Biswas, "Detection of coronavirus disease (covid-19) based on deep features and support vector machine," 2020.

[143] J. Zhao, Y. Zhang, X. He, and P. Xie, "Covid-ct-dataset: a ct scan dataset about covid-19," arXiv preprint arXiv:2003.13865, vol. 490 , 2020.

[144] D. Das, K. Santosh, and U. Pal, "Truncated inception net: Covid19 outbreak screening using chest x-rays," Physical and engineering sciences in medicine, vol. 43, no. 3, pp. 915-925, 2020.

[145] "Covid-19 resources," https://www.bsti.org.uk/covid-19-resources/, accessed: 2021-06-5

[146] "Normal chest imaging examples," https://radiopaedia.org/articles/ normal-chest-imaging-examples?lang=gb, accessed: 2021-06-5.

[147] K. He, X. Zhang, S. Ren, and J. Sun, "Deep residual learning for image recognition," in Proceedings of the IEEE conference on computer vision and pattern recognition, 2016, pp. 770-778.
[148] A. Abbas, M. M. Abdelsamea, and M. M. Gaber, "Classification of covid-19 in chest X-ray images using detrac deep convolutional neural network," arXiv preprint arXiv:2003.13815, 2020.

[149] S. Hassantabar, M. Ahmadi, and A. Sharifi, "Diagnosis and detection of infected tissue of covid-19 patients based on lung X-ray image using convolutional neural network approaches," Chaos, Solitons \& Fractals, vol. 140 , p. $110170,2020$.

[150] M. Sandler, A. Howard, M. Zhu, A. Zhmoginov, and L.-C. Chen, "Mobilenetv2: Inverted residuals and linear bottlenecks," in Proceedings of the IEEE conference on computer vision and pattern recognition, 2018 , pp. 4510-4520.

[151] A. A. Ardakani, A. R. Kanafi, U. R. Acharya, N. Khadem, and A. Mohammadi, "Application of deep learning technique to manage covid-19 in routine clinical practice using ct images: Results of 10 convolutional neural networks," Computers in biology and medicine, vol. 121, p. 103795, 2020.

[152] M. Bolhassani, "Transfer learning approach to classify the X-ray image that corresponds to corona disease using resnet50 pretrained by chexnet," arXiv preprint arXiv:2105.08382, 2021

[153] A. Jaiswal, N. Gianchandani, D. Singh, V. Kumar, and M. Kaur, "Classification of the covid-19 infected patients using densenet201 based deep transfer learning," Journal of Biomolecular Structure and Dynamics, pp. 1-8, 2020

[154] K. Adam, I. I. Mohamed, and Y. Ibrahim, "A selective mitigation technique of soft errors for dnn models used in healthcare applications: Densenet201 case study," IEEE Access, vol. 9, pp. 65803-65823, 2021.

[155] N. Dey, Y.-D. Zhang, V. Rajinikanth, R. Pugalenthi, and N. S. M. Raja, "Customized vgg19 architecture for pneumonia detection in chest Xrays," Pattern Recognition Letters, vol. 143, pp. 67-74, 2021.

[156] C. Szegedy, V. Vanhoucke, S. Ioffe, J. Shlens, and Z. Wojna, "Rethinking the inception architecture for computer vision," in Proceedings of the IEEE conference on computer vision and pattern recognition, 2016 , pp. 2818-2826.

[157] Y. LeCun, K. Kavukcuoglu, and C. Farabet, "Convolutional networks and applications in vision," in Proceedings of 2010 IEEE international symposium on circuits and systems. IEEE, 2010, pp. 253-256.

[158] A. ResNet and I. VGGNet, "Understanding various architectures of convolutional networks," 2019.

[159] Delta variant: What we know about the science. [Online]. Available: https://www.cdc.gov/coronavirus/2019-ncov/variants/delta-variant.htm

[160] P. P. Ray, "A survey on internet of things architectures," Journal of King Saud University-Computer and Information Sciences, vol. 30, no. 3, pp. 291-319, 2018.

[161] J. Lin, W. Yu, N. Zhang, X. Yang, H. Zhang, and W. Zhao, "A survey on internet of things: Architecture, enabling technologies, security and privacy, and applications," IEEE Internet of Things Journal, vol. 4 no. 5, pp. 1125-1142, 2017.

[162] W. Yu, F. Liang, X. He, W. G. Hatcher, C. Lu, J. Lin, and X. Yang, "A survey on the edge computing for the internet of things," IEEE access, vol. 6, pp. 6900-6919, 2017.

[163] How smart, connected products are transforming competition. [Online]. Available: https://hbr.org/2014/11/ how-smart-connected-products-are-transforming-competition

[164] Rfid and iot in a smart hospital: benefits and challenges of smart patient tracking. [Online]. Available: https://www.scnsoft.com/blog/ rfid-and-iot-in-a-smart-hospital

[165] V. Jagadeeswari, V. Subramaniyaswamy, R. Logesh, and V. Vijayakumar, "A study on medical internet of things and big data in personalized healthcare system," Health information science and systems, vol. 6 , no. 1 , pp. 1-20, 2018

[166] F. Ali, S. El-Sappagh, S. R. Islam, A. Ali, M. Attique, M. Imran, and K.-S. Kwak, "An intelligent healthcare monitoring framework using wearable sensors and social networking data," Future Generation Computer Systems, vol. 114, pp. 23-43, 2021.

[167] M. E. Chowdhury, K. Alzoubi, A. Khandakar, R. Khallifa R. Abouhasera, S. Koubaa, R. Ahmed, and A. Hasan, "Wearable real-time heart attack detection and warning system to reduce road accidents," Sensors, vol. 19, no. 12, p. 2780, 2019.

[168] A. Gurjar and N. A. Sarnaik, "Heart attack detection by heartbeat sensing using internet of things: Iot," Heart, vol. 5, no. 03, 2018.

[169] D. R. Seshadri, E. V. Davies, E. R. Harlow, J. J. Hsu, S. C. Knighton, T. A. Walker, J. E. Voos, and C. K. Drummond, "Wearable sensors for covid-19: a call to action to harness our digital infrastructure for remote patient monitoring and virtual assessments," Frontiers in Digital Health, vol. 2, p. 8, 2020. 
[170] M. Romano, T. Onorati, I. Aedo, and P. Diaz, "Designing mobile applications for emergency response: citizens acting as human sensors," Sensors, vol. 16, no. 3, p. 406, 2016.

[171] M. M. Islam, S. Mahmud, L. Muhammad, M. R. Islam, S. Nooruddin, and S. I. Ayon, "Wearable technology to assist the patients infected with novel coronavirus (covid-19)," SN Computer Science, vol. 1, no. 6, pp. 1-9, 2020.

[172] H. C. Ates, A. K. Yetisen, F. Güder, and C. Dincer, "Wearable devices for the detection of covid-19," Nature Electronics, vol. 4, no. 1, pp. 13-14, 2021

[173] N. Al Bassam, S. A. Hussain, A. Al Qaraghuli, J. Khan, E. Sumesh, and V. Lavanya, "Iot based wearable device to monitor the signs of quarantined remote patients of covid-19," Informatics in Medicine Unlocked, vol. 24, p. 100588, 2021.

[174] V. Nathan and R. Jafari, "Particle filtering and sensor fusion for robust heart rate monitoring using wearable sensors," IEEE journal of biomedical and health informatics, vol. 22, no. 6, pp. 1834-1846, 2017.

[175] T. Arakawa, "Recent research and developing trends of wearable sensors for detecting blood pressure," Sensors, vol. 18, no. 9, p. 2772 2018 .

[176] A. Henriksen, M. H. Mikalsen, A. Z. Woldaregay, M. Muzny, G. Hartvigsen, L. A. Hopstock, and S. Grimsgaard, "Using fitness trackers and smartwatches to measure physical activity in research: analysis of consumer wrist-worn wearables," Journal of medical Internet research, vol. 20, no. 3, p. e9157, 2018.

[177] H. Thapliyal, V. Khalus, and C. Labrado, "Stress detection and management: A survey of wearable smart health devices," IEEE Consumer Electronics Magazine, vol. 6, no. 4, pp. 64-69, 2017.

[178] S. Choi et al., "Dealing with menstrual pain and difficulties: holistic experiences using a heating wearable device for management of menstrual pain," 2018.

[179] R. Jain et al., "Doctors' perceptions on the use of internet of things medical devices (iot-mds) for anemic pregnant women: A tam2 study," International Journal of Healthcare Information Systems and Informatics (IJHISI), vol. 16, no. 1, pp. 58-80, 2021.

[180] K. Grym, H. Niela-Vilén, E. Ekholm, L. Hamari, I. Azimi, A. Rahmani, P. Liljeberg, E. Löyttyniemi, and A. Axelin, "Feasibility of smart wristbands for continuous monitoring during pregnancy and one month after birth," BMC pregnancy and childbirth, vol. 19, no. 1, pp. 1-9, 2019.

[181] I. Azimi, T. Pahikkala, A. M. Rahmani, H. Niela-Vilén, A. Axelin, and P. Liljeberg, "Missing data resilient decision-making for healthcare iot through personalization: A case study on maternal health," Future Generation Computer Systems, vol. 96, pp. 297-308, 2019.

[182] Garmin: Hrm-tri. [Online]. Available: https://buy.garmin.com/en-US/ US/p/136403

[183] Polar: H7 heart rate sensor. [Online]. Available: https://support.polar. com/en/support/H7_heart_rate_sensor

[184] Pure pulse heart rate. [Online]. Available: https://www.fitbit.com/ global/us/technology/heart-rate

[185] Tomtom spark cardio. [Online]. Available: https://www.tomtom.com/ en_sg/sports/fitness-watches/gps-watch-cardio-spark/

[186] C.-W. Cho, W.-H. Chao, S.-H. Lin, and Y.-Y. Chen, "A visionbased analysis system for gait recognition in patients with parkinson's disease," Expert Systems with applications, vol. 36, no. 3, pp. 7033 7039, 2009.

[187] F. Casamassima, A. Ferrari, B. Milosevic, P. Ginis, E. Farella, and L. Rocchi, "A wearable system for gait training in subjects with parkinson's disease," Sensors, vol. 14, no. 4, pp. 6229-6246, 2014.

[188] Department uses thermal imaging to detect covid-19. [Online]. Available: https://www.defense.gov/Explore/News/Article/Article/2178320/ department-uses-thermal-imaging-to-detect-covid-19/

[189] Baidu : Top artificial intelligence innovations from the chinese 'google'. [Online]. Available: https://www.analyticsinsight.net/ baidu-top-artificial-intelligence-innovations-from-the-chinese-google/

[190] S. S. Vedaei, A. Fotovvat, M. R. Mohebbian, G. M. Rahman, K. A. Wahid, P. Babyn, H. R. Marateb, M. Mansourian, and R. Sami, "Covid-safe: An iot-based system for automated health monitoring and surveillance in post-pandemic life," IEEE Access, vol. 8, pp. 188538 188551,2020

[191] R. Sami, F. Soltaninejad, B. Amra, Z. Naderi, S. Haghjooy Javanmard, B. Iraj, S. Haji Ahmadi, A. Shayganfar, M. Dehghan, N. Khademi et al., "A one-year hospital-based prospective covid-19 open-cohort in the eastern mediterranean region: The khorshid covid cohort (kcc) study," PloS one, vol. 15, no. 11, p. e0241537, 2020
[192] D. Gupta, S. Bhatt, M. Gupta, and A. S. Tosun, "Future smart connected communities to fight covid-19 outbreak," Internet of Things, p. 100342 , 2020 .

[193] K. Kumar, N. Kumar, and R. Shah, "Role of iot to avoid spreading of covid-19," International Journal of Intelligent Networks, vol. 1, pp. 32-35, 2020.

[194] L. L. Wang, K. Lo, Y. Chandrasekhar, R. Reas, J. Yang, D. Eide, K. Funk, R. Kinney, Z. Liu, W. Merrill et al., "Cord-19: The covid-19 open research dataset," ArXiv, 2020.

[195] A. Roy, F. H. Kumbhar, H. S. Dhillon, N. Saxena, S. Y. Shin, and S. Singh, "Efficient monitoring and contact tracing for covid-19: A smart iot-based framework," IEEE Internet of Things Magazine, vol. 3, no. 3, pp. 17-23, 2020.

[196] Draganfly selected to globally integrate breakthrough health diagnosis technology immediately onto autonomous camera's and specialized drones to combat coronavirus and future health emergencies. [Online]. Available: https://www.globenewswire.com/news-release/ Draganfly-Selected-to-Globally-Integrate-Breakthrough

[197] Drones and the coronavirus: The many ways drones are supporting containment efforts in china. [Online]. Available: https://uavcoach com/drones-coronavirus/

[198] Drone technology: A new ally in the fight against covid-19. [Online]. Available: https://www.mdlinx.com/ article/drone-technology-a-new-ally-in-the-fight-against-covid-19/ 61gQW7xmBCg6LyGy43925Z

[199] Spain's military uses dji agricultural drones to spray disinfectant in fight against covid-19. [Online]. Available: https://www.scmp.com/tech/gear/article/3077945/ spains-military-uses-dji-agricultural-drones-spray-disinfectant-fight 2

[200] Delhi civic body begins thermal screening people on balconies with drones. [Online]. Available: https://www.ndtv.com/delhi-news/ coronavirus-delhi-civic-body-using-drones

[201] Drones to stop the covid-19 epidemic. [Online]. Available: https: //www.bbva.com/en/drones-to-stop-the-covid-19-epidemic/

[202] A. Kumar, K. Sharma, H. Singh, S. G. Naugriya, S. S. Gill, and R. Buyya, "A drone-based networked system and methods for combating coronavirus disease (covid-19) pandemic," Future Generation Computer Systems, vol. 115, pp. 1-19, 2021

[203] D. M. El-Din, A. E. Hassanein, E. E. Hassanien, and W. M. Hussein, "E-quarantine: A smart health system for monitoring coronavirus patients for remotely quarantine," arXiv preprint arXiv:2005.04187, 2020.

[204] S. A. Fatima, N. Hussain, A. Balouch, I. Rustam, M. Saleem, and M. Asif, "Iot enabled smart monitoring of coronavirus empowered with fuzzy inference system," Int. J. Adv. Res. Ideas Innov. Technol, vol. 6 , no. $1,2020$.

[205] Clinical management of severe acute respiratory infection (sari) when covid-19 disease is suspected. [Online]. Available: https://www.who.int/docs/default-source/coronaviruse/ clinical-management-of-novel-cov.pdf

[206] Managing the respiratory care of patients with covid-19. [Online]. Available: http://www.sipirs.it/cms/wp-content/uploads/2020/ 03/Managing-the-Respiratory-care-of-patients-with-COVID-19.pdf

[207] D. J. Miller, J. V. Capodilupo, M. Lastella, C. Sargent, G. D. Roach, V. H. Lee, and E. R. Capodilupo, "Analyzing changes in respiratory rate to predict the risk of covid-19 infection," PLoS One, vol. 15, no. 12 , p. e0243693, 2020.

[208] Y. Nam, B. A. Reyes, and K. H. Chon, "Estimation of respiratory rates using the built-in microphone of a smartphone or headset," IEEE journal of biomedical and health informatics, vol. 20, no. 6, pp. 1493$1501,2015$.

[209] C. Massaroni, A. Nicolò, D. Lo Presti, M. Sacchetti, S. Silvestri, and E. Schena, "Contact-based methods for measuring respiratory rate," Sensors, vol. 19, no. 4, p. 908, 2019.

[210] C. Massaroni, J. Di Tocco, M. Bravi, A. Carnevale, D. L. Presti, R. Sabbadini, S. Miccinilli, S. Sterzi, D. Formica, and E. Schena, "Respiratory monitoring during physical activities with a multi-sensor smart garment and related algorithms," IEEE Sensors Journal, vol. 20, no. 4, pp. 2173-2180, 2019.

[211] C. Song, P. Zeng, Z. Wang, H. Zhao, and H. Yu, "Wearable continuous body temperature measurement using multiple artificial neural networks," IEEE Transactions on Industrial Informatics, vol. 14, no. 10, pp. 4395-4406, 2018.

[212] J.-W. Lin, M.-H. Lu, and Y.-H. Lin, "A thermal camera based continuous body temperature measurement system," in Proceedings of the IEEE/CVF International Conference on Computer Vision Workshops, 2019, pp. 0-0. 
[213] N. A. Zakaria, F. N. B. M. Saleh, and M. A. A. Razak, "Iot (internet of things) based infant body temperature monitoring," in 2018 2nd international conference on biosignal analysis, processing and systems (ICBAPS). IEEE, 2018, pp. 148-153.

[214] C. Kulkarni, H. Karhade, S. Gupta, P. Bhende, and S. Bhandare, "Health companion device using iot and wearable computing," in 2016 International Conference on Internet of Things and Applications (IOTA). IEEE, 2016, pp. 152-156.

[215] M. F. Farah and Z. B. Ramadan, "Disruptions versus more disruptions: How the amazon dash button is altering consumer buying patterns," Journal of Retailing and Consumer Services, vol. 39, pp. 54-61, 2017.

[216] The new aws iot button can control your car, other home devices. [Online]. Available: https://techseen.com/aws-iot-button/

[217] P. R. Chai, H. Zhang, C. W. Baugh, G. D. Jambaulikar, J. C. McCabe, J. M. Gorman, E. W. Boyer, and A. Landman, "Internet of things buttons for real-time notifications in hospital operations: proposal for hospital implementation," Journal of medical Internet research, vol. 20, no. 8, p. e251, 2018.

[218] A. Gohil, H. Modi, and S. K. Patel, "5g technology of mobile communication: A survey," in 2013 international conference on intelligent systems and signal processing (ISSP). IEEE, 2013, pp. 288-292.

[219] S. Latif, J. Qadir, S. Farooq, and M. A. Imran, "How 5g wireless (and concomitant technologies) will revolutionize healthcare?" Future Internet, vol. 9, no. 4, p. 93, 2017. 